\title{
Dopamine Transporter Localization in Medial Forebrain Bundle Axons Indicates Its Long-Range Transport Primarily by Membrane Diffusion with a Limited Contribution of Vesicular Traffic on Retromer-Positive Compartments
}

\author{
${ }^{\circledR}$ Tarique R. Bagalkot, ${ }^{1}$ Ethan R. Block, ${ }^{1,3}$ Kristen Bucchin, ${ }^{2}$ Judith Joyce Balcita-Pedicino, ${ }^{2}$ Michael Calderon, ${ }^{1}$ \\ Susan R. Sesack, ${ }^{2}$ and ${ }^{-}$Alexander Sorkin ${ }^{1}$ \\ ${ }^{1}$ Department of Cell Biology, University of Pittsburgh School of Medicine, Pittsburgh, Pennsylvania $15261,{ }^{2}$ Department of Neuroscience, Dietrich \\ School of Arts and Sciences, University of Pittsburgh, Pittsburgh, Pennsylvania 15213, and ${ }^{3}$ Chatham University, Pittsburgh, Pennsylvania 15232
}

\begin{abstract}
Dopamine transporter (DAT) controls dopamine neurotransmission by clearing synaptically released dopamine. However, trafficking itineraries of DAT, which determine its cell-surface concentration near synapses, are poorly characterized. It is especially unknown how DAT is transported between spatially distant midbrain somatodendritic and striatal axonal compartments. To examine this "long-range" trafficking, the localization and membrane diffusion of HA-epitope tagged DAT in the medial forebrain bundle (MFB) of a knock-in mouse (both sexes) were analyzed using confocal, super-resolution and EM in intact brain and acute brain slices. HA-DAT was abundant in the plasma membrane of MFB axons, similar to the striatum, although the intracellular fraction of HA-DAT in MFB was more substantial. Intracellular HA-DAT colocalized with VPS35, a subunit of the retromer complex mediating recycling from endosomes, in a subset of axons. Late endosomes, lysosomes, and endoplasmic reticulum were abundant in the soma but minimally present in MFB axons, suggesting that biosynthesis and lysosomal degradation of DAT are confined to soma. Together, the data suggest that membrane diffusion is the main mode of long-range DAT transport through MFB, although the contribution of vesicular traffic can be significant in a population of MFB axons. Based on HA-DAT diffusion rates, plasma membrane DAT in MFB axons turns over with a halftime of $\sim 20 \mathrm{~d}$, which explains the extremely slow turnover of DAT protein in the brain. Unexpectedly, the mean diameter of DAT-labeled MFB axons was observed to be twice larger than reported for striatum. The implications of this finding for dopamine neuron physiology are discussed.
\end{abstract}

Key words: dopamine transporter; EM; mouse brain; trafficking

Significance Statement

The dopamine transporter (DAT) is a key regulator of dopamine neurotransmission and a target of abused psychostimulants. In the present study, we examined, for the first time, mechanisms of the long-range traffic of DAT in intact brain and acute brain slices from the knock-in mouse expressing epitope-tagged DAT. Using a combination of confocal, super-resolution and EM, we defined DAT localization and its membrane diffusion parameters in medial forebrain bundle axonal tracts connecting midbrain somatodendritic and striatal axonal compartments of dopaminergic neurons. In contrast to the widely accepted model of long-range axonal transport, our studies suggest that DAT traffics between midbrain and striatum, mainly by lateral diffusion in the plasma membrane with only a limited contribution of vesicular transport in recycling endosomes.

Received Mar. 31, 2020; revised Nov. 5, 2020; accepted Nov. 13, 2020.

Author contributions: T.R.B., E.R.B., S.R.S., and A.S. designed research; T.R.B., E.R.B., K.B., J.J.B.-P., and M.

C. performed research; T.R.B., E.R.B., K.B., M.C., S.R.S., and A.S. analyzed data; T.R.B. wrote the first draft of the paper; T.R.B., E.R.B., S.R.S., and A.S. edited the paper; E.R.B., S.R.S., and A.S. wrote the paper.

This work was supported by National Institutes of Health/National Institute on Drug Abuse Grant DA014204 to A.S., S.R.S., and E.R.B. We thank Dr. Boris Slepchenko (University of Connecticut) for help in the FRAP data analysis; and Dr. Robert Edwards (UCSF School of Medicine) and Dr. M. N. Rasband (Baylor College of Medicine) for gifts of antibodies.

The authors declare no competing financial interests.

Correspondence should be addressed to Susan R. Sesack at sesack@pitt.edu or Alexander Sorkin at sorkin@pitt.edu.

https://doi.org/10.1523/JNEUROSCI.0744-20.2020

Copyright $(2021$ the authors

\section{Introduction}

Dopamine (DA) signaling plays a major role in modulating cognition, locomotion, motivation, and reward-seeking behaviors (Giros and Caron, 1993; Dani and Zhou, 2004). Dysregulation of DA signaling is linked to addiction and neuropsychiatric disorders, including Parkinson's disease, schizophrenia, attention-deficit/hyperactivity disorder, and autism spectrum disorder (Snyder, 2002; Gainetdinov and Caron, 2003; Iversen and Iversen, 2007; Wise, 2008; Gowrishankar et al., 2014; Volkow and Morales, 2015). Dopaminergic neurons in SNc and VTA project to the dorsal striatum (dStr), NAc, and 
frontal cortex, forming nigrostriatal, mesolimbic, and mesocortical pathways, respectively (Ciliax et al., 1999). Striatal DA axons are arborized extensively, with each branch having numerous varicosities containing active zones, mitochondria, and synaptic vesicles (Nirenberg et al., 1996; Hersch et al., 1997; Matsuda et al., 2009). The branched axonal network is connected to midbrain soma by minimally arborized axonal "tracts" within the medial forebrain bundle (MFB), which spans 3-4 mm in rat brain (Matsuda et al., 2009). This subcompartment has not been well characterized, and the mechanisms of "long-range" transport of organelles and macromolecules between striatum and soma are not known.

Mechanisms of long-range axonal transport have been studied extensively in DRGs and other peripheral neurons, with a substantive literature on dynein-mediated retrograde transport of early and late endosomes in cultured neurons (Surana et al., 2020). Anterograde axonal transport of transmembrane proteins synthesized in the ER and post-translationally modified in the Golgi apparatus in the soma is mediated by kinesin-dependent microtubular transport (Surana et al., 2020). Additionally, local translation of membrane proteins in axonal termini, including transmembrane proteins, such as Robo3.2 and CD166 antigen, is an emerging mechanism for the regulation of synaptic protein turnover (Colak et al., 2013; Jung et al., 2014; González et al., 2016; Y. Wu et al., 2017; Luarte et al., 2018). However, evidence for rough ER in mature CNS axons is sparse, and conventional Golgi apparatus has not been demonstrated in presynaptic areas of the intact brain (Luarte et al., 2018).

Plasma membrane DA reuptake transporter (DAT) controls the duration and intensity of DA neurotransmission (Giros et al., 1991, 1996; Jaber et al., 1997). DAT plasma membrane density and proximity to release sites are therefore critical for regulation of DA neurotransmission. The itineraries and mechanisms of axonal trafficking of DAT in intact dopaminergic neurons have not been well defined, and how optimal concentrations of DAT are maintained near synaptic areas remains unknown. EM studies of rodent dopaminergic axons and immunofluorescence analyses of acute brain slices have demonstrated that DAT is predominantly located in extrasynaptic areas of the plasma membrane, with a small pool of intracellular DAT, suggesting minimal constitutive trafficking of DAT in the NAc and dStr (Nirenberg et al., 1996, 1997; Hersch et al., 1997; Ciliax et al., 1999; Block et al., 2015). A relatively large fraction of DAT is detected in endosomes, ER, and Golgi in midbrain soma and proximal dendrites, indicative of more extensive DAT trafficking in these compartments (Nirenberg et al., 1996, 1997; Hersch et al., 1997; Ciliax et al., 1999; Block et al., 2015). However, the kinetics and mechanisms of transport of newly synthesized DAT from midbrain to striatal synapses are unknown. Furthermore, whether and how axonal DAT, destined for degradation, is delivered back to the somatodendritic compartment remain to be elucidated.

In this study, we analyzed the localization and membrane diffusion parameters of DAT within MFB axonal tracts of dopaminergic neurons of a knock-in mouse that expresses DAT tagged with an extracellular hemagglutinin epitope (HA11) (Rao et al., 2012). Immunofluorescence imaging and immunogold labeling with EM demonstrated a relatively small pool of DAT in intracellular compartments and predominant plasma membrane localization of DAT in MFB axons. We propose that lateral diffusion in the plasma membrane is the main means of DAT bidirectional transport between midbrain and striatum, whereas the contribution of vesicular transport is significant only in a limited population of neurons.

\section{Materials and Methods}

Animals. HA-DAT knock-in mice (on the C57BL/6J background) were maintained in $12 \mathrm{~h}$ light/dark cycle at constant temperature and humidity. Food and water were available ad libitum. All procedures were conducted in accordance with the National Institutes of Health's Guide for the care and use of laboratory animals and with the approval of the Institutional Animal Care and Use Committee of the University of Pittsburgh. Mice of both sexes were used in all experiments.

Reagents. Antibodies were purchased from the following sources: mouse monoclonal antibodies against the HA11 epitope (16B12) were from BioLegend (mms-101p); rabbit monoclonal anti-HA11 were from Cell Signaling (3724S); rat monoclonal anti-lysosome-associated membrane protein 1 (LAMP1) antibodies were from the University of Iowa Developmental Studies Hybridoma Bank (1D4B) (Chen et al., 1985); and rabbit polyclonal antibodies against early endosomal antigen 1 (EEA1) (ab2900), CD63 (ab216130), and Rab5 (ab13253) were from Abcam. Rabbit and goat polyclonal antibodies against vacuolar protein sorting-associated protein 35 (VPS35) were from Thermo Fisher Scientific (PA5-21898) and NovusBio (NB100-1397), respectively. Rabbit polyclonal antibody to vesicular monoamine transporter 2 (VMAT2) was kindly provided by Robert Edwards (UCSF School of Medicine). Rabbit polyclonal KDEL antibody was from Invitrogen (PA1-013), and rabbit polyclonal anti-reticulon 3 (RTN3) antibody was from Millipore (ABN1723). Rabbit polyclonal $\beta$-IV spectrin antibody was kindly provided by M. N. Rasband (Baylor College of Medicine). Normal rabbit IgG was from Santa Cruz Biotechnology (sc2027). Rat IgG2a $\kappa$ Iso Control was from eBioscience (14-4321-82). Secondary donkey anti-mouse, anti-rat, and anti-rabbit AffiniPure antibodies conjugated with AlexaFluor-488 (A488), Cy5, or Cy3 were from Jackson ImmunoResearch Laboratories, and gold-conjugated secondary antibodies were from Aurion (800.022). A488-conjugated goat anti-mouse Fab fragment antibodies (115-547-003) and Cy3-conjugated goat anti-mouse Fc $\gamma$-specific Fab fragment antibodies were from Jackson ImmunoResearch Laboratories (115-167-185). Secondary goat anti-mouse and anti-rabbit AffiniPure antibodies conjugated with AlexaFluor-568 (A11004) and AlexaFluor-660 (A21074) were from Invitrogen. All other reagents and supplies were from Thermo Fisher Scientific unless noted otherwise.

Immunofluorescence staining of cryosections. Mice of both sexes were anesthetized with xylazine/ketamine and subject to transcardial perfusion with ice-cold PBS followed by freshly prepared 4\% PFA in PBS. Brains were gently removed and postfixed in the same fixative for $4 \mathrm{~h}$, and then rinsed with PBS and cryoprotected by incubating in $20 \%$ sucrose in $\mathrm{PBS}$ at $4^{\circ} \mathrm{C}$ overnight or until brains sank to the bottom of the tube, followed by $30 \%$ sucrose in PBS at $4^{\circ} \mathrm{C}$ until ready for cryosectioning. Immediately before cutting, brains were embedded in OCT Tissue-Tek compound (Sakura Finetek) and deep frozen in liquid nitrogen. Tissue was sectioned at $50 \mu \mathrm{m}$ with a CM3050 cryostat (Leica Microsystems). Free-floating cryosections were washed and then treated to inhibit endogenous peroxidase by $10 \mathrm{~min}$ incubation in $1 \%$ hydrogen peroxide. Tissue was then washed, permeabilized with $0.1 \%$ Triton-X-100 for $1 \mathrm{~h}$, and preincubated with blocking buffer containing 10\% normal donkey serum (D9663, Sigma Millipore), 3\% BSA (A2153, Sigma Millipore), 0.1\% Triton X-100 (Sigma Millipore) in PBS for $1 \mathrm{~h}$ at room temperature followed by incubation with primary antibodies at $4^{\circ} \mathrm{C}$ in PBS containing $10 \%$ normal donkey serum, $0.1 \%$ Triton X-100, and 3\% BSA for $48 \mathrm{~h}$. Primary antibodies were diluted to $1: 1000$, except for $\beta$-IV spectrin (1:400) unless indicated otherwise. After three washes in PBS, sections were incubated with corresponding secondary antibodies for $1 \mathrm{~h}$ at room temperature, or $24 \mathrm{~h}$ for $\beta$-IV spectrin. Nuclei were stained with Hoechst 33342 (62249, Thermo Fisher Scientific). Sections were mounted with Prolong gold antifade mounting medium (P36930, Thermo Fisher Scientific).

Spinning disk confocal microscopy. To obtain high-resolution threedimensional (3D) images of DA neurons in brain slices, a $z$ stack of 
confocal images was acquired using a spinning-disk confocal system based on an Axio Observer Z1 inverted fluorescence microscope (Carl Zeiss) equipped with a $63 \times$ Plan Apo PH, 1.4 NA objective, computer-controlled Spherical Aberration Correction unit, Yokogawa CSU-W1, Vector photomanipulation module, Photometrics Evolve 16-bit EMCCD and Hamamatsu Orca-Flash4.0 CMOS cameras, environmental chamber, piezo stage controller and lasers (405, 445, 488, 515,561 , and $640 \mathrm{~nm}$ ), all controlled by SlideBook 6 software (Intelligent Imaging Innovations). Flash4.0 was used for imaging of fixed samples, and Evolve was used in experiments with living brain slices. Typically, 10-30 serial two-dimensional (2D) confocal images of cryosections were recorded at $400 \mathrm{~nm}$ intervals. Colocalization of HA-DAT with endocytic markers was visually evaluated by identifying clear overlapping structures that could be followed in multiple $z$ planes.

The length of axon initial segments (AISs) and the distances of the proximal ends of AIS from the soma were measured in HA-DAT-labeled neurons costained with $\beta$-IV spectrin using the SlideBook ruler tool. To measure the diameter of HA-DAT-labeled axons outside or within the AIS, a segment mask was generated to select voxels containing HA-DAT fluorescence. Ten measurements of the axon width in the confocal section from the $3 \mathrm{D}$ image stack that displayed a maximal cross-section were made, and mean values for each individual axon were calculated. In the case of AIS, 10 measurements of the diameter were made throughout the entire length of AIS, from its proximal to distal ends at identical length intervals between measurements.

Quantification of colocalization. A $z$ stack of $x-y$ confocal double-labeled images were cropped to generate new images consisting of a $z$ stack of three consecutive confocal sections with maximally strong labeling and similar penetration of both antibodies, typically mouse or rabbit HA11 against HA-DAT (488 $\mathrm{nm}$ channel fluorescence) and an organelle marker or VMAT2 (640 nm channel fluorescence). These images were deconvolved using the no-neighbor algorithm of SlideBook6. Background-subtracted images were then used to generate an automated segmentation Mask \#1 to select voxels positive for $488 \mathrm{~nm}$ channel fluorescence and colocalization Mask \#2 (common voxels positive for $488 \mathrm{~nm}$ and $640 \mathrm{~nm}$ channel fluorescence) using an Interactive Segmentation tool of SlideBook6 and the same minimal fluorescence threshold as in Mask \#1. The fraction of HA-DAT (488 nm channel) colocalized with an organelle marker ( $640 \mathrm{~nm}$ channel) of the total HA-DAT was calculated as the ratio of integrated fluorescence intensities of the $488 \mathrm{~nm}$ channel in Mask \#2 to that of Mask \#1 in each 3D image containing multiple axons.

To compare the fraction of HA-DAT and VMAT2 colocalized with VPS35 in dopaminergic axons, colocalization was quantified on cryosections colabeled with all three antibodies. Three-plane 3D images were generated and deconvolved as above for double-labeled images. The fraction of HA-DAT ( $488 \mathrm{~nm}$ channel) colocalized with VPS35 $(640 \mathrm{~nm}$ channel) was calculated as described above for double-labeled images. Total fluorescence of VMAT2 (561 nm channel) in dopaminergic axons was quantified by generating Mask \#1-561 containing voxels common for 561 and $488 \mathrm{~nm}$ channel fluorescence (HA-DAT). Colocalization mask of VMAT2 and VPS35 in dopaminergic neurons was generated by selecting voxels positive for 561,488 , and $640 \mathrm{~nm}$ channel fluorescence (Mask \#2-561) with the minimal fluorescence thresholds of 488 and $640 \mathrm{~nm}$ channel fluorescence to be identical to those thresholds used for calculating the HA-DAT/VPS35 colocalization in the same image. The fraction of VMAT2 colocalized with VPS35 of the total VMAT2 fluorescence in dopaminergic neurons was then calculated as the ratio of integrated fluorescence intensities of the $561 \mathrm{~nm}$ channel in Mask \#2-561 to that in Mask \#1-561 in each image containing multiple axons.

Preparation of acute brain slices. Procedures followed were similar to those described previously (Block et al., 2015). Six- to 8-week old HADAT mice were killed by isoflurane inhalation followed by decapitation. Brains were removed and submerged into an ice slush of oxygenated (by bubbling with carbogen, $95 \% \mathrm{O}_{2}$ and $5 \% \mathrm{CO}_{2}$ ) cutting-specific ACSF (in mM) as follows: $87 \mathrm{NaCl}, 2.5 \mathrm{KCl}, 1.25 \mathrm{NaH}_{2} \mathrm{PO}_{4}, 25.7 \mathrm{NaHCO}_{3}, 7.0$ $\mathrm{MgSO}_{4}, 0.5 \mathrm{CaCl}_{2}, 25$ dextrose, 75 sucrose, 0.15 ascorbic acid, 1.0 kynurenic acid). Microtome blades were used to make sagittal cuts in a stainless-steel slicing block along the midline and at 0.8 and $1.6 \mathrm{~mm}$ lateral. Slices were allowed to recover in carbogen-bubbled normal ACSF
(nACSF, in mM) as follows: $124 \mathrm{NaCl}, 4 \mathrm{KCl}, 1.25 \mathrm{NaH}_{2} \mathrm{PO}_{4}, 25.7$ $\mathrm{NaHCO}_{3}, 1.2 \mathrm{MgSO}_{4}, 2.45 \mathrm{CaCl}_{2}, 11$ dextrose, 0.15 ascorbic acid) for $30 \mathrm{~min}$ at room temperature.

Fluorescence recovery after photobleaching (FRAP) analysis. For labeling of HA-DAT, slices were incubated in $0.5 \mathrm{ml} \mathrm{nACSF}$ at room temperature with $10 \mu \mathrm{g} / \mathrm{ml}$ anti-HA antibodies for $30 \mathrm{~min}$, adding $0.1 \mathrm{ml}$ freshly oxygenated nACSF every $5 \mathrm{~min}$. Unbound HA antibodies were removed by incubation for $20 \mathrm{~min}$ in oxygenated nACSF. Slices were then incubated in $0.5 \mathrm{ml} \mathrm{nACSF}$ at room temperature with $5 \mu \mathrm{g} / \mathrm{ml}$ A488-conjugated goat anti-mouse Fab fragment antibodies for $30 \mathrm{~min}$, adding $0.1 \mathrm{ml}$ freshly oxygenated nACSF every $5 \mathrm{~min}$. Unbound antibodies were removed, and slices were equilibrated to FRAP assay temperature by incubation for at least $20 \mathrm{~min}$ in oxygenated nACSF at room temperature or $37^{\circ} \mathrm{C}$

To image living dopaminergic neurons, acute sagittal brain slices were transferred to MatTek dishes containing oxygenated nACSF and placed within the microscope's $37^{\circ} \mathrm{C}$ environmental chamber. Images were acquired using the spinning disk confocal imaging system described above. Typically, 50 time-lapse images were collected at 3-5 s intervals from a single confocal plane $10 \mu \mathrm{m}$ deep from the cut face of the slice. Photobleaching was performed on user-selected regions (axonal length of $\sim 6.35 \mu \mathrm{m}$ ) using $80 \%$ laser power for $3 \mathrm{~ms}$. Regions were chosen based on a lack of crowding with other HA-DAT-expressing structures and a continuity of signal intensity and focus on either side of the bleached region. Regions from images with stable focus and minimal sample lateral movement were analyzed using the "FRAP" module in SlideBook6 software to obtain values of $\tau_{\mathrm{D}}$ (recovery half-time) and mobile fraction ( $M f$, or fraction recovered). To account for image-wide photobleaching during the duration of the experiment, signal intensities of analyzed regions were normalized over time to those of an unbleached region. The diffusion coefficient $D$ was calculated as described previously (Blasius et al., 2013). Briefly, the axon in a FRAP experiment can be modeled as a cylinder where the length of the bleached region is $2 \mathrm{a}$. The cylindrical symmetry reduces the model to a one-dimensional (1D) problem where concentration (or fluorescence intensity) can be described as a function of time $(t)$ and distance $(x)$ along the axon. Diffusion coefficient was calculated using Equation 1: $D=\left(\mathrm{a} / x_{1 / 2}\right)^{2} / \tau_{\mathrm{D}}$ where a value of $x_{1 / 2}$ was determined to be $\sim 1.03,975$ by plotting the function $g(x)$ in the range covering the value $g\left(x_{1 / 2}\right)=0.5$. To estimate the half-time of DAT turnover in MFB axons $(\sim 1.5-2.5 \mathrm{~mm}$ in length in mouse brain), a "photobleaching" model was used, in which the "photobleached" region was considered to be $2 \mathrm{~mm}$ (2a) and recovery occurred from both sides, the soma and the striatal complex, assuming unlimited source of DAT in both of these regions. Therefore, Equation 1 is converted to Equation 2 as follows: $\tau_{\mathrm{D}}=\left(\mathrm{a} / x_{1 / 2}\right)^{2} / D$, where $\mathrm{a}=1 \mathrm{~mm}$.

To examine potential endocytosis of HA-DAT/antibody complexes during FRAP experiments at $37^{\circ} \mathrm{C}$, acute slices were incubated with mouse HA11 antibodies and Cy3-conjugated Fab fragment of goat antimouse Fc-specific antibodies $(2.5 \mu \mathrm{g} / \mathrm{ml})$ at room temperature as described above. After subsequent $40 \mathrm{~min}$ incubation at $37^{\circ} \mathrm{C}$, slices were incubated in nACSF with $5 \mu \mathrm{g} / \mathrm{ml}$ A488-conjugated Fab fragment of secondary goat anti-mouse-IgG antibodies (that is not Fc-specific) at $4^{\circ} \mathrm{C}$ for $30 \mathrm{~min}$ to label exclusively cell-surface HA11:HA-DAT complexes. After washing 3 times with $4^{\circ} \mathrm{C}$ nACSF, slices were fixed in $4 \%$ PFA for $30 \mathrm{~min}$ at $4^{\circ} \mathrm{C}$. $3 \mathrm{D}$ imaging was performed using a spinning disk confocal system, and colocalization of $488 \mathrm{~nm}$ (plasma membrane HADAT) and $561 \mathrm{~nm}$ (total surface plus internalized HA-DAT) channel fluorescence was estimated by calculating Pearson correlation coefficient using SlideBook6.

Stimulated emission depletion (STED) super-resolution 3D microscopy. Free-floating sagittal sections were obtained using a cryostat, permeabilized as mentioned above, and labeled with antibodies against HADAT and VPS35, followed by fluorophore-conjugated secondary antibodies. HA-DAT STED imaging was performed using a TCS SP8 super resolution STED microscope (Leica Microsystems) with a pulsed white light laser and an AOBS detection system (Leica Microsystems). Images were collected using the $775 \mathrm{~nm}$ STED laser line with 40\% 3D STED using the Leica Microsystems STED objective (HC PL APO 100×/ NA1.40 oil) with a $200 \mathrm{~Hz}$ scan speed and $6 \times$ line averaging. Pixel size 


\section{A HA-DAT/EEA1/nuclei}
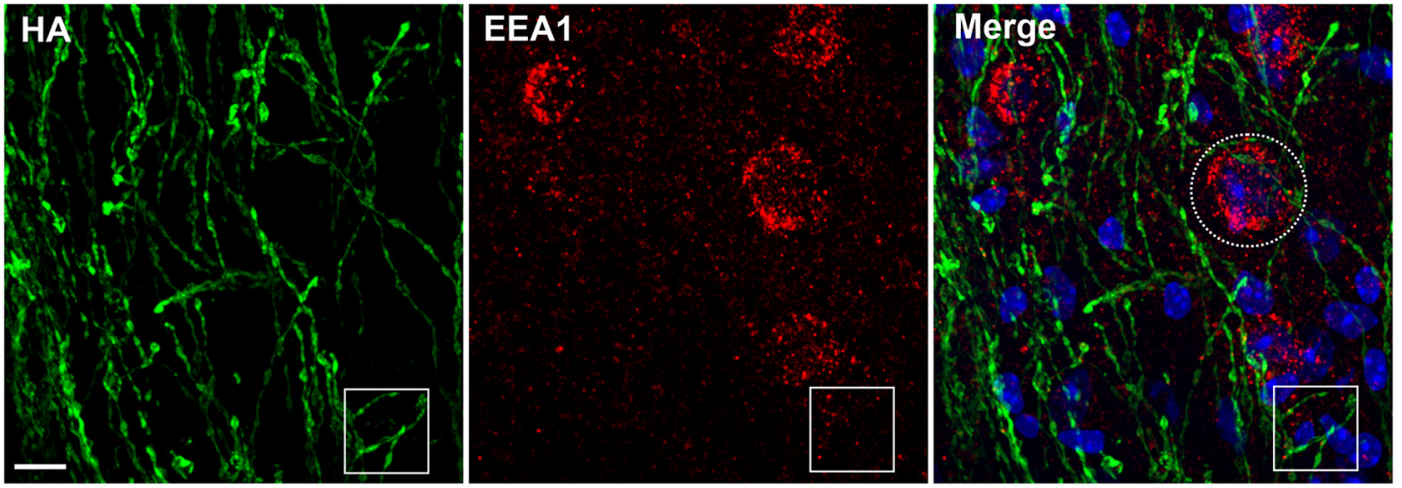

B
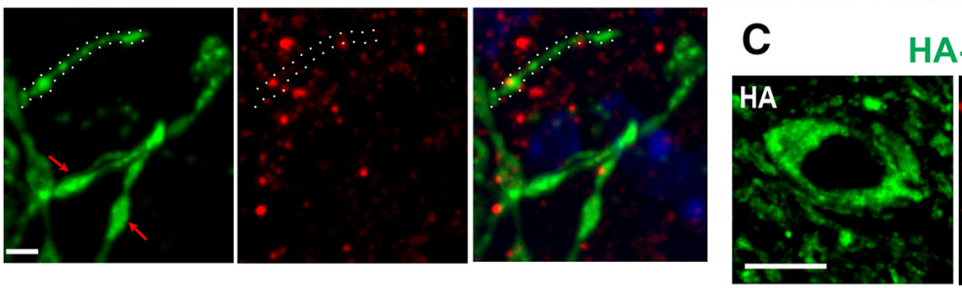

HA-DAT/EEA1/nuclei
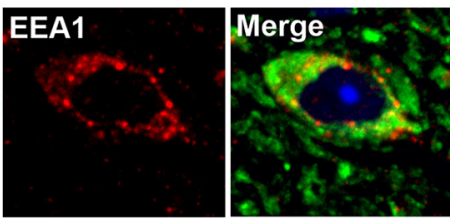

D HA-DAT/RAB5/nuclei
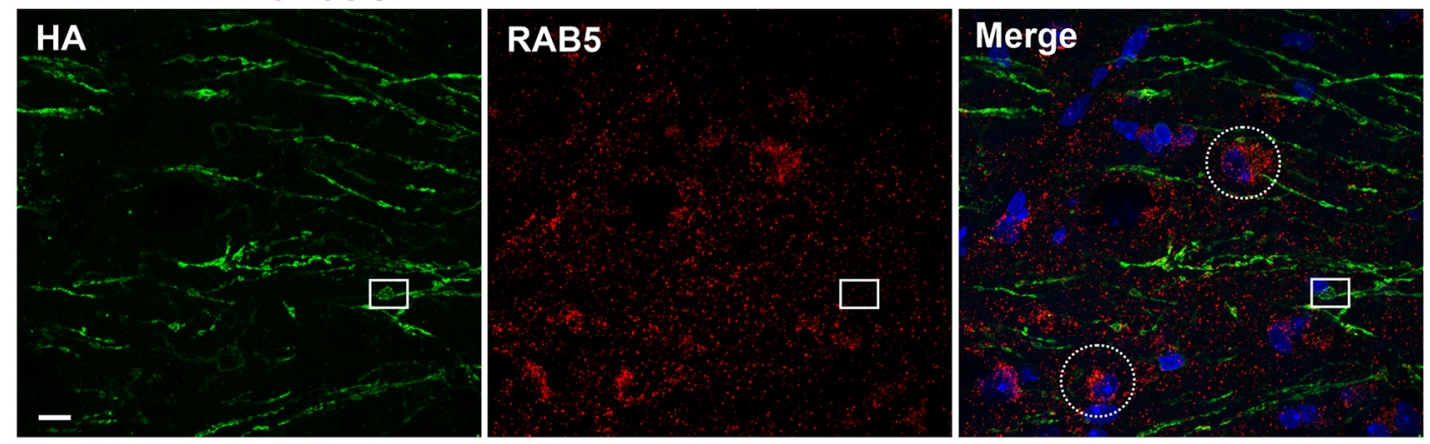

$\mathbf{E}$

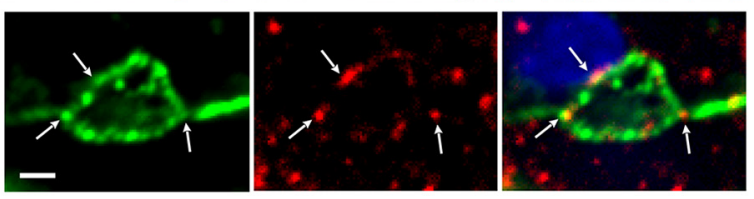

G

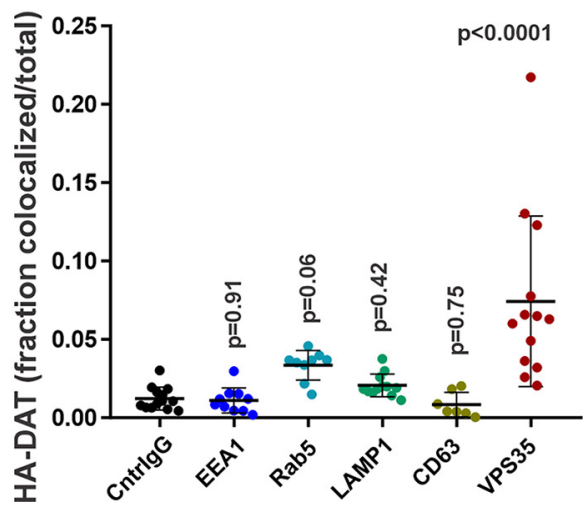

Figure 1. Coimmunolabeling of HA-DAT with EEA1 and Rab5 in MFB axons and soma of dopaminergic axons. Brains were fixed by cardiac perfusion with PFA and prepared for sectioning as described in Materials and Methods. Sagittal cryosections were labeled with antibodies against HA and EEA1 $(\boldsymbol{A}-\boldsymbol{C})$ or Rab5 $(\boldsymbol{D}-\boldsymbol{F})$ followed by fluorophore-conjugated secondary antibodies. 3D stacks of confocal images were acquired through $640 \mathrm{~nm}$ (red, EEA1 or Rab5), $488 \mathrm{~nm}$ (green, HA-DAT), and $405 \mathrm{~nm}$ (blue, Hoechst) channels from MFB $(\boldsymbol{A}, \boldsymbol{B}, \boldsymbol{D}, \boldsymbol{E})$ and midbrain $(\boldsymbol{C}, \boldsymbol{F})$ regions. Maximum intensity projections of three consecutive $x$-y confocal sections are shown. $\boldsymbol{B}, \boldsymbol{E}$, Insets, High-magnification images corresponding to white rectangles in $\boldsymbol{A}$ and $\boldsymbol{D}$, respectively. $\boldsymbol{B}$, Red arrows indicate varicosities in the axon. White punctate lines indicate boundaries of representative axons. $\boldsymbol{E}$, White arrows indicate examples of HA-DAT/Rab5 colocalization in axonal varicosity. $A, D$, White circles represent examples of characteristic perinuclear EEA1 and Rab5 labeling in HA-DAT-negative (nondopaminergic) cells. C, $F, 0$ verlap of EEA.1 and Rab5 with HA-DAT in the soma of dopaminergic cells. Scale bars: $\boldsymbol{A}, \boldsymbol{C}, \boldsymbol{D}, \boldsymbol{F}, 10 \mu \mathrm{m} ; \boldsymbol{B}, \boldsymbol{E}, 2 \mu \mathrm{m}$. $\boldsymbol{G}$, Quantification of the fraction of HA-DAT colocalized with endosome/lysosome markers from images generated as in $\boldsymbol{A}$ and $\boldsymbol{D}$, and images of HA-DAT colabeling with LAMP1, CD63, and VPS35 generated as in Figures 2 and 3. Cryosections labeled with normal rabbit or rat lgGs used at the same concentrations $(2 \mu \mathrm{g} / \mathrm{ml})$ and imaged as corresponding specific rabbit or rat lgGs against endolysosomal markers were used as control for nonspecific overlap of the fluorescence signals. Scatter dot plot represents mean values with SDs ( $n=8-13$ images depicting multiple axons). $p$ values were calculated against control lgGs using one-way ANOVA followed by Fisher's LSD multiple comparison test.

was set to $19 \mathrm{~nm} /$ pixel; step size was set to $1.50 \mu \mathrm{m}$, and pinhole was set at $87.3 \mu \mathrm{m}(0.575 \mathrm{AU})$. HA-DAT was visualized using AlexaFluor-568, exciting at $575 \mathrm{~nm}$ and detecting between 588 and $657 \mathrm{~nm}$ and temporally gated between 1.01 and $5.01 \mathrm{~ns}$. VPS35 was visualized with
AlexaFluor-660, exciting at $663 \mathrm{~nm}$ and detecting between $672 \mathrm{~nm}$ and $758 \mathrm{~nm}$ and temporally gated between 1.0 and $6.0 \mathrm{~ns}$.

Labeling of HA-DAT in vivo by stereotactic injections of HA11 antibodies. Eight- to 10-week-old HA-DAT mice of either sex were used. 


\section{A HA-DAT/LAMP1/nuclei}
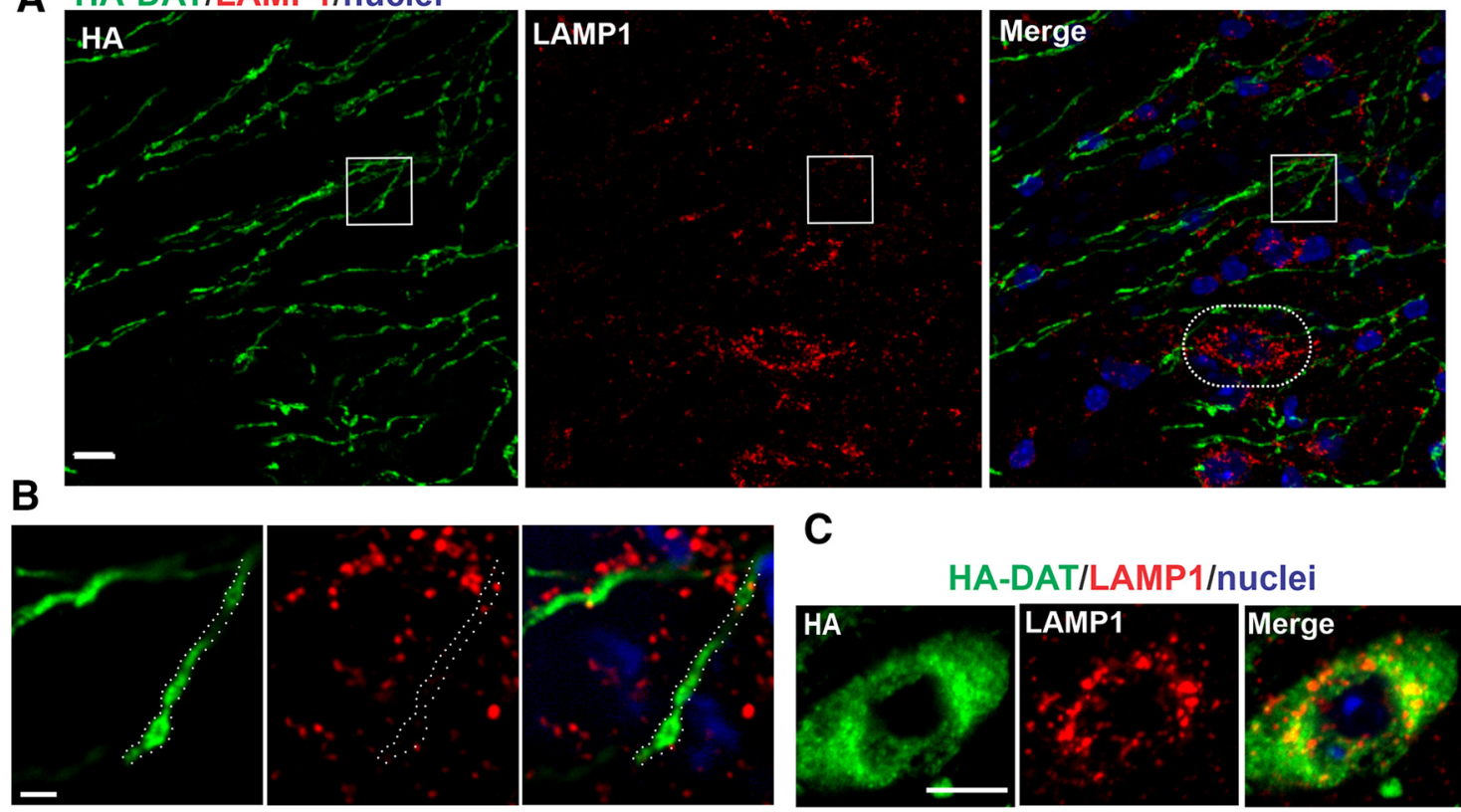

\section{HA-DAT/CD63/nuclei}

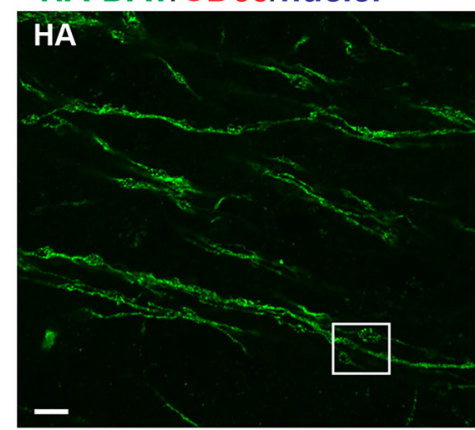

E

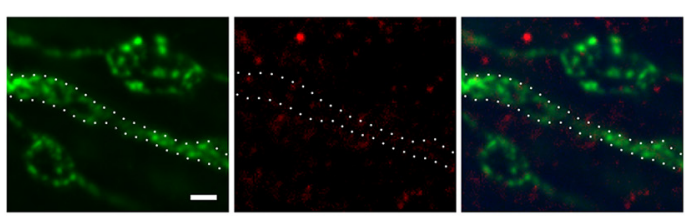

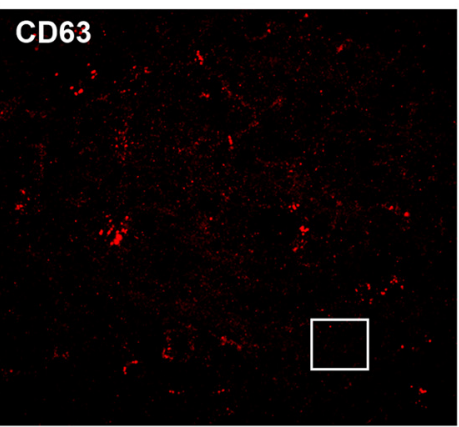

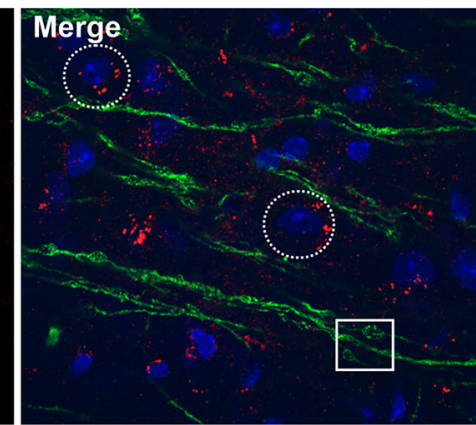

$\mathbf{F}$

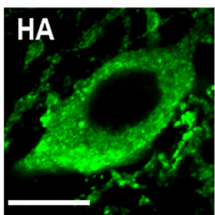

HA-DAT/CD63/nuclei

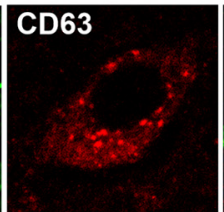

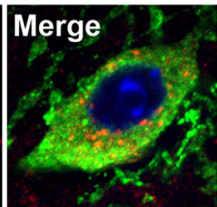

Figure 2. Coimmunolabeling of HA-DAT with LAMP1 and CD63 in MFB axons and soma of dopaminergic axons. Brains were fixed by cardiac perfusion with PFA and prepared for sectioning as described in Materials and Methods. Sagittal cryosections were colabeled with rabbit or mouse HA11 antibodies, and antibodies against LAMP1 (A-C) or CD63 (D-F), respectively, followed by fluorophore-conjugated secondary antibodies. 3D stacks of confocal images were acquired through $640 \mathrm{~nm}$ (red, LAMP1 and (D63), $488 \mathrm{~nm}$ (green, HA-DAT), and $405 \mathrm{~nm}$ (blue, Hoechst) channels from MFB $(\boldsymbol{A}, \boldsymbol{B}, \boldsymbol{D}, \boldsymbol{E})$ and midbrain $(\boldsymbol{C}, \boldsymbol{F})$ regions. $\boldsymbol{B}, \boldsymbol{E}$, Insets, High-magnification images corresponding to white rectangles in $\boldsymbol{A}$ and $\boldsymbol{D}$, respectively. White punctate lines indicate boundaries of representative axons. All images are maximum intensity projections of three consecutive $x-y$ confocal sections, except in $B$ (inset) where a single confocal section is shown. Examples of characteristic perinuclear LAMP1 and CD63 labeling in HA-DAT-negative (nondopaminergic) cells are indicated by white oval and circles in $\boldsymbol{A}$ and $\boldsymbol{D}$. LAMP1 and CD63 colabeling with HA-DAT in the soma of dopaminergic cells is illustrated in $\boldsymbol{C}$ and $\boldsymbol{F}$, respectively. Scale bars: $\boldsymbol{A}, \boldsymbol{C}, \boldsymbol{D}, \boldsymbol{F}, 10 \mu \mathrm{m} ; \boldsymbol{B}, \boldsymbol{E}, 2 \mu \mathrm{m}$.

Anesthesia was induced with intraperitoneal injection of xylazine/ketamine. Mice were placed in a digitalized stereotactic frame with nontraumatic ear bars (Stoelting) equipped with a mouse adaptor. A dental drill was used to create two bilateral holes over MFB. Using a $5 \mu$ Hamilton syringe, $5 \mu$ l of mouse HA.11 antibody (final concentration $0.1 \mu \mathrm{g} / \mu \mathrm{l}$ in PBS) was injected bilaterally directly into the MFB (coordinates relative to bregma, $\mathrm{AP}= \pm 1.5 \mathrm{~mm} ; \mathrm{ML}= \pm 1.5 \mathrm{~mm} ; \mathrm{DV}=-5.2 \mathrm{~mm}$ ), according to Paxinos and Watson (1998) at the rate of $1 \mu \mathrm{l} / \mathrm{min}$, and the injection needle was left in place for $10 \mathrm{~min}$ before retraction to allow proper diffusion and to prevent reflux of the antibody. Mice were kept on a warm hotpad for $\sim 2 \mathrm{~h}$ after surgery and then killed using isoflurane, followed by decapitation. Brains were removed and after an initial sagittal cut along the midline, 0.8 -mm-thick slices were made using microtome blades and a stainless-steel slicing block. Slices were fixed for $1 \mathrm{~h}$ with freshly prepared $4 \%$ PFA, followed by incubation with the secondary A488-conjugated donkey anti-mouse-IgG antibody in PBS at $4^{\circ} \mathrm{C}$ overnight to label HA-DAT on the surface of neurons. Slices were then washed with PBS, permeabilized with $0.3 \%$ Triton X-100, and incubated with rabbit monoclonal HA11 antibody (to label all HA-DAT not occupied by mouse HA11) and goat polyclonal VPS35 antibody in PBS at $4^{\circ} \mathrm{C}$ overnight. Secondary donkey anti-rabbit-IgG and anti-goat-IgG AffiniPure antibodies conjugated with $\mathrm{Cy} 3$ and $\mathrm{Cy} 5$, respectively, were applied for $1 \mathrm{~h}$ at room temperature. Nuclei were stained with Hoechst 33342. Sections were imaged in $35 \mathrm{~mm}$ MatTek dishes through 488, 561, and $640 \mathrm{~nm}$ (VPS35) channels using spinning disk confocal microscope at least $5 \mu \mathrm{m}$ deep from the surface of the slice as described above. 


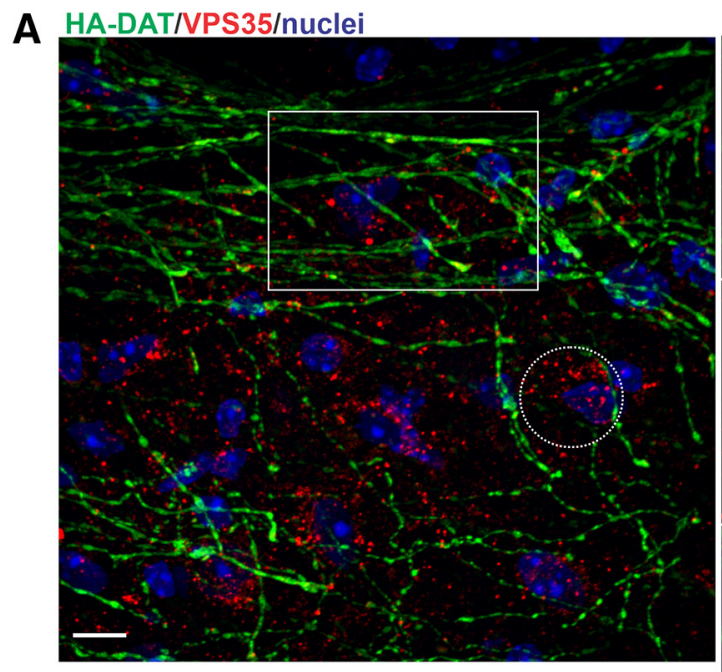

\section{B}
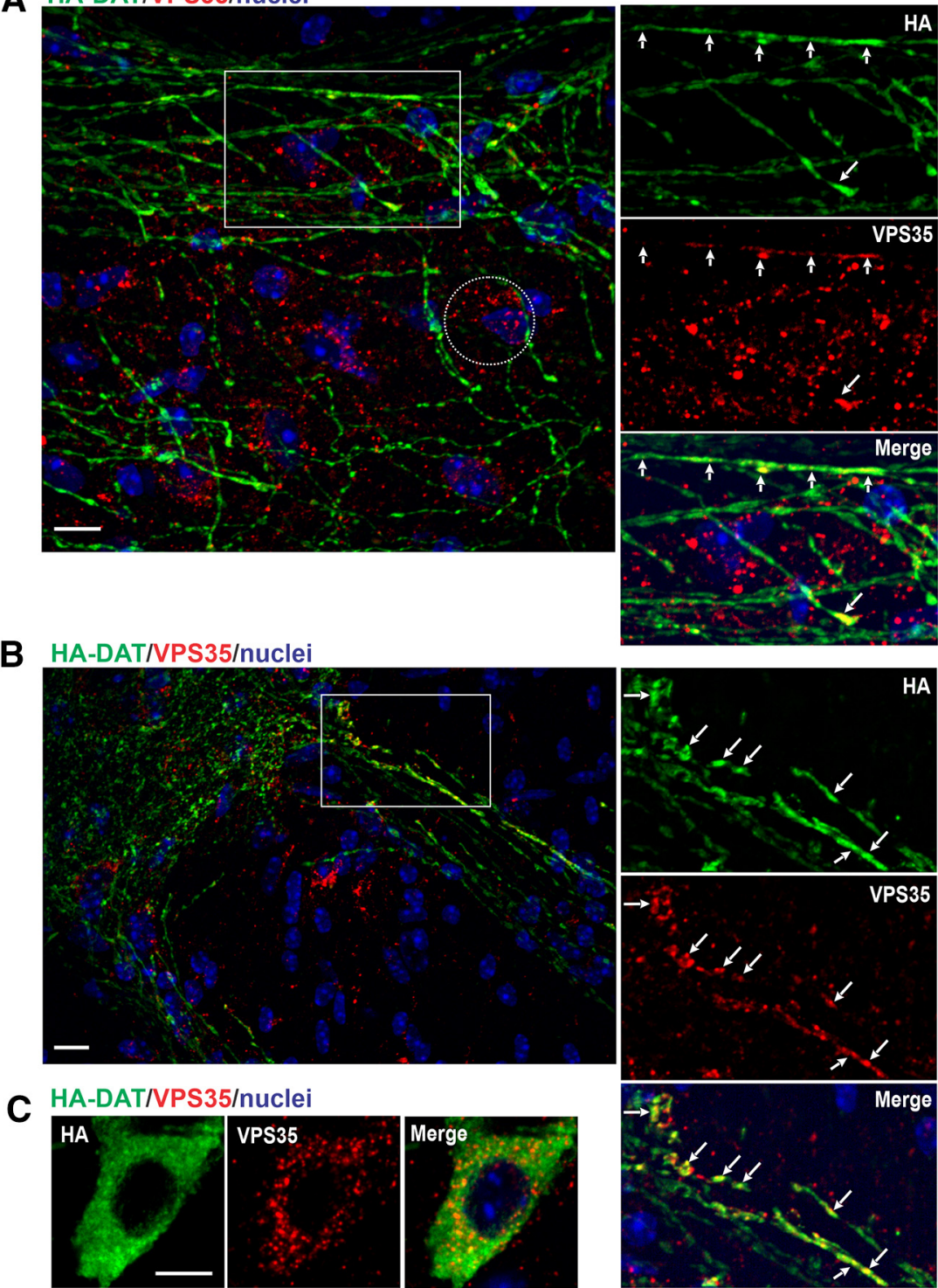

Figure 3. Coimmunolabeling of HA-DAT and VPS35 in MFB axons and soma of dopaminergic axons. Brains were fixed by cardiac perfusion with PFA and prepared for sectioning as described in Materials and Methods. Sagittal cryosections were labeled with antibodies against VPS35 and HA11 followed by fluorophore-conjugated secondary antibodies. 3D stacks of images of MFB $(\boldsymbol{A})$, region just caudal to striatum $(\boldsymbol{B})$, and midbrain $(\boldsymbol{C})$ were acquired using a spinning disk confocal system through $640 \mathrm{~nm}$ (red, VPS35), $488 \mathrm{~nm}$ (green, HA-DAT), and $405 \mathrm{~nm}$ (blue, Hoechst) channels. Maximum intensity projections of three consecutive $x-y$ confocal sections are shown. Insets, Split-channel images of areas indicated by white rectangles. Arrows indicate examples of HA-DAT and VPS35 colocalization. $\boldsymbol{A}$, White circles represent examples of characteristic VPS35 labeling in HA-DAT-negative (nondopaminergic) cells. C, Typical distribution of VPS35 endosomes in dopaminergic neuron soma. Scale bars, $10 \mu \mathrm{m}$.

Colocalization of $488 \mathrm{~nm}$ (plasma membrane HA-DAT) and $561 \mathrm{~nm}$ (total HA-DAT) channel fluorescence was estimated by calculating Pearson correlation coefficient using SlideBook6.

EM. Four HA-DAT mice were used for EM examination of the nigrostriatal portion of the MFB. Two of the subjects were control animals from our prior investigation of the effects of amphetamine (Block et al., 2015) and were acutely injected intraperitoneally with saline before being killed $1 \mathrm{~h}$ later. The other 2 subjects were naive. All mice were anesthetized with pentobarbital (60-100 mg/kg i.p.) and then administered the zinc chelator diethyl-dithiocarbamate $(1 \mathrm{~g} / \mathrm{kg}$ i.p.) for $5-15 \mathrm{~min}$ before death to prevent artifactual silver binding to endogenous zinc (Veznedaroglu and Milner, 1992). Animals were then perfused intracardially with $5-10 \mathrm{ml}$ of heparin-saline $100 \mathrm{U} / \mathrm{ml}$, followed by $50-100 \mathrm{ml}$ of $1 \%$ glutaraldehyde, and 4\% PFA in $0.1 \mathrm{M} \mathrm{PB}, \mathrm{pH}$ 7.4. Brains were then removed from the skull, kept in fixative for 30$60 \mathrm{~min}$, and then transferred to $\mathrm{PB}$ before being sectioned at $50 \mu \mathrm{m}$ on a vibratome.

Sections were treated with $1 \%$ sodium borohydride in PB for $30 \mathrm{~min}$ and then rinsed in $\mathrm{PB}$. Tissue was then transferred to a cryoprotectant before being subjected to freezing at $-80^{\circ} \mathrm{C}$ and thawing in room temperature $\mathrm{PB}$. The cryoprotectant was then rinsed, and the sections were transferred to 0.1 M TBS, pH 7.6. Tissue was then incubated for $30 \mathrm{~min}$ in blocking solution containing 3\% normal donkey serum, $1 \%$ BSA, and $0.04 \%$ Triton X-100 in TBS. Afterwards, primary monoclonal anti-HA (HA11) antibody was added to this solution, and the tissue was incubated for $\sim 36 \mathrm{~h}$ at $4^{\circ} \mathrm{C}$. Sections were then rinsed thoroughly in TBS before being transferred to $0.01 \mathrm{M}$ PBS, pH 7.4. Another blocking solution was then used to incubate the tissue for $30 \mathrm{~min}$ : 3\% normal donkey serum, $0.8 \%$ BSA, and $0.1 \%$ coldwater fishskin gelatin (Aurion) in PBS. To this solution was added 1:50 goat anti-mouse IgG conjugated to gold particles sized $0.8 \mathrm{~nm}$ (Aurion). Tissue remained in secondary antibody overnight before being rinsed thoroughly in blocking solution followed by PBS. Sections were treated for $10 \mathrm{~min}$ in $2.5 \%$ glutaraldehyde in PBS and rinsed again. Sections were then incubated in 1:10 proprietary enhanced conditioning solution (Aurion) before placement in R-Gent SE-EM reagents (Aurion) for 1-2 h. After the silver enhancement of bound gold, tissue was rinsed again in 1:10 enhanced conditioning solution before being transferred to PB.

To prepare tissue for EM examination, sections were treated with $1 \%$ osmium tetroxide in $\mathrm{PB}$ for $30 \mathrm{~min}$ to fix lipids and then rinsed in PB. Tissue was then dehydrated in increasing concentrations of ethanol followed by propylene oxide. Incubation in epoxy resin began with an overnight treatment in 1:1 propylene oxide and Embed-812 (Electron Microscopy Sciences) followed by straight epoxy resin for 2-3 h. Sections were then cured for $72 \mathrm{~h}$ at $60^{\circ}$ $\mathrm{C}$ between commercial plastic sheets kept flat by heavy weights. Regions of the MFB that contained the densest concentration of dopaminergic axons were identified in these sections by light microscopic comparison with tissue from WT mice labeled by immunoperoxidase for TH. These regions were photographed before being cut out and annealed to blocks of solid epoxy resin. The tissue was trimmed to a trapezoid shape containing the ROI, and ultrathin sections were sliced from the surface of this region at $60 \mathrm{~nm}$. Tissue collected onto copper 400 mesh grids was then contraststained with uranyl acetate and lead citrate and examined in an FEI Morgagni TEM.

The nigrostriatal portion of the MFB was identified at low magnification as areas of clustered axons containing immunogold-silver labeling for HA-DAT. Such areas could only be seen near the surface of the tissue at the interface with plastic resin, as antibodies typically do not penetrate well below this depth. These regions were then analyzed at 18,000$28,000 \times$ and photographed when axons with specific immunoreactivity were encountered; these were defined as containing at least three immunogold particles on the membrane or 5 particles in total. Additional photomicrographs were taken from the same regions as well as immediately 
adjacent fields to estimate nonspecific labeling in profiles comparably sized to axons labeled for HA-DAT. Digital micrographs were imported into MicroBrightField Neurolucida program version 9, and profiles were then traced and analyzed for area and perimeter. Diameter was determined manually from the micrographs as the straight line across the widest part of the short axis (Sesack et al., 1998). Using a calibrated cursor, gold particles were categorized as membrane-associated when they were within $20 \mathrm{~nm}$ of the plasmalemma. Other gold particles were considered intracellular, and those within $20 \mathrm{~nm}$ of organelles were recorded. Circular organelles $\sim 40-50 \mathrm{~nm}$ in diameter were considered to be vesicles; larger organelles were measured for area and perimeter. Assuming a relatively circular shape, organelle diameter was then estimated as $2 \times$ the square root of area $/ \pi$.

Statistical analysis. All statistical analyses were performed using GraphPad Prism software (GraphPad). For comparisons of each two groups, unpaired Student's $t$ test was used after verification of equal variance using $F$ test. Welch's correction was performed when the variance across groups was assumed to be unequal. For multiple comparison analyses, a one-way ANOVA followed by Tukey's or Fisher's LSD multiple comparison tests was used. All experiments were performed at least three times. Differences were considered significant when the $p$ value was $<0.05$, with the specific $p$ values detailed within each figure legend.

\section{Results}

Immunofluorescence analysis of HA-DAT localization in endosomes and lysosomes in MFB axons

Sagittal sectioning and spinning disk confocal microscopy enabled imaging of the entire length of axonal tracts, from the midbrain through the MFB to the region caudal to striatum. HA-DAT was found to be distributed along the entire length of axons that displayed randomly dispersed widenings and varicosities (Fig. $1 A, B$ ). HA-DAT fluorescence was seen mostly as an uninterrupted continuous signal along large parts of these axons, although clusters of HA-DAT were frequently observed (Fig. 1A,B). To test whether clustered HA-DAT fluorescence represented cell-surface clusters or intracellular vesicles, such as endosomes or lysosomes, HA-DAT was coimmunolabeled with the resident proteins of these compartments.

Compartments containing EEA1, a marker of early and sorting endosomes, were not detected in MFB axonal tracts labeled with the HA11 antibody (Fig. 1A,B). Similarly, Rab5, a small GTPase enriched in all types of early endosomes and in newly formed endocytic vesicles, was largely excluded from HA-DAT-labeled MFB axons (Fig. 1D,E). Rarely, small puncta of colocalized HA-DAT and Rab5 were observed in axonal varicosities (Fig. $1 E)$. At the same time, characteristic punctate distribution of EEA1 and Rab5 was observed clearly in neighboring cells surrounding MFB axons (Fig. $1 A, D$ ) and in dopaminergic neuronal soma in the midbrain (Fig. $1 C, F$ ), thus confirming the specificity and the sensitivity of EEA1 and Rab5 immunolabeling. Quantifications demonstrated that the fraction of HA-DAT colocalized with EEA.1 and Rab5 in MFB axons was small and comparable with such fraction resulting from nonspecific signal overlap measured using normal IgG (Fig. 1G). Consistent with the occasional

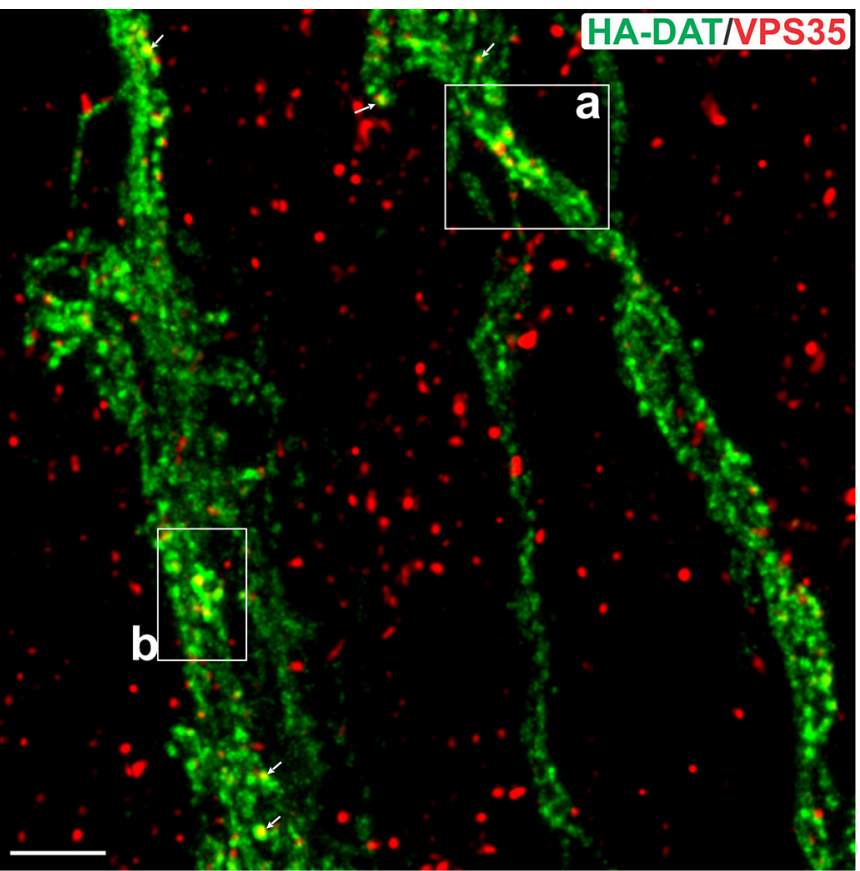

a
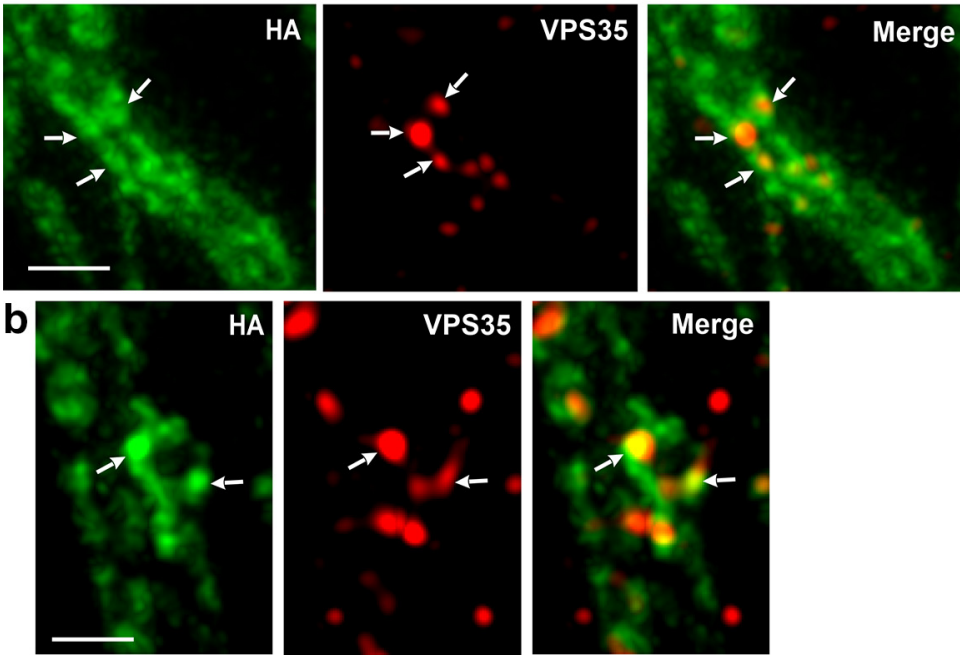

4. STED imaging of HA-DAT and VPS35 in MFB axons. Brains were fixed by cardiac perfusion with PFA and prepared for sectioning as described in Materials and Methods. Sagittal cryosections were labeled with antibodies against VPS35 and HA11 followed by fluorophore-conjugated secondary antibodies. 3D stacks of $x$-y jections of three consecutive $x$-y optical sections (merged channels) are shown. $\boldsymbol{a}, \boldsymbol{b}$ Insets, High-magnification, split-channel images of areas indicated by white rectangles. Arrows indicate examples of HA-DAT and VPS35 colocalization. Scale bars: Insets, $\boldsymbol{a}, 3 \mu \mathrm{m} ; \boldsymbol{b}, 1 \mu \mathrm{m}$.

detection of Rab5/HA-DAT compartments (Fig. 1E), quantifications suggested a trend for specific colocalization of Rab5 and HADAT in a few images (Fig. $1 G$ ). However, this colocalization was not statistically significant (Fig. $1 G$ ). Late endosomes and lysosomes, labeled with LAMP1 and CD63, were also not unambiguously detected along MFB axonal tracts labeled with HA11 antibody (Fig. $2 A, B, D, E)$, whereas these compartments exhibited a characteristic pattern of perinuclear distribution in cells surrounding MFB axons (Fig. $2 A, D$ ) and in the soma of dopaminergic neurons (Fig. 2C,F). Individual LAMP1 puncta were seldomly seen overlapping with dopaminergic axons. However, staining with nonspecific normal IgG also resulted in randomly distributed puncta that occasionally overlapped with the HA11 labeling. Fractions of HA-DAT colocalized with LAMP1 and CD63 were extremely small and not different 

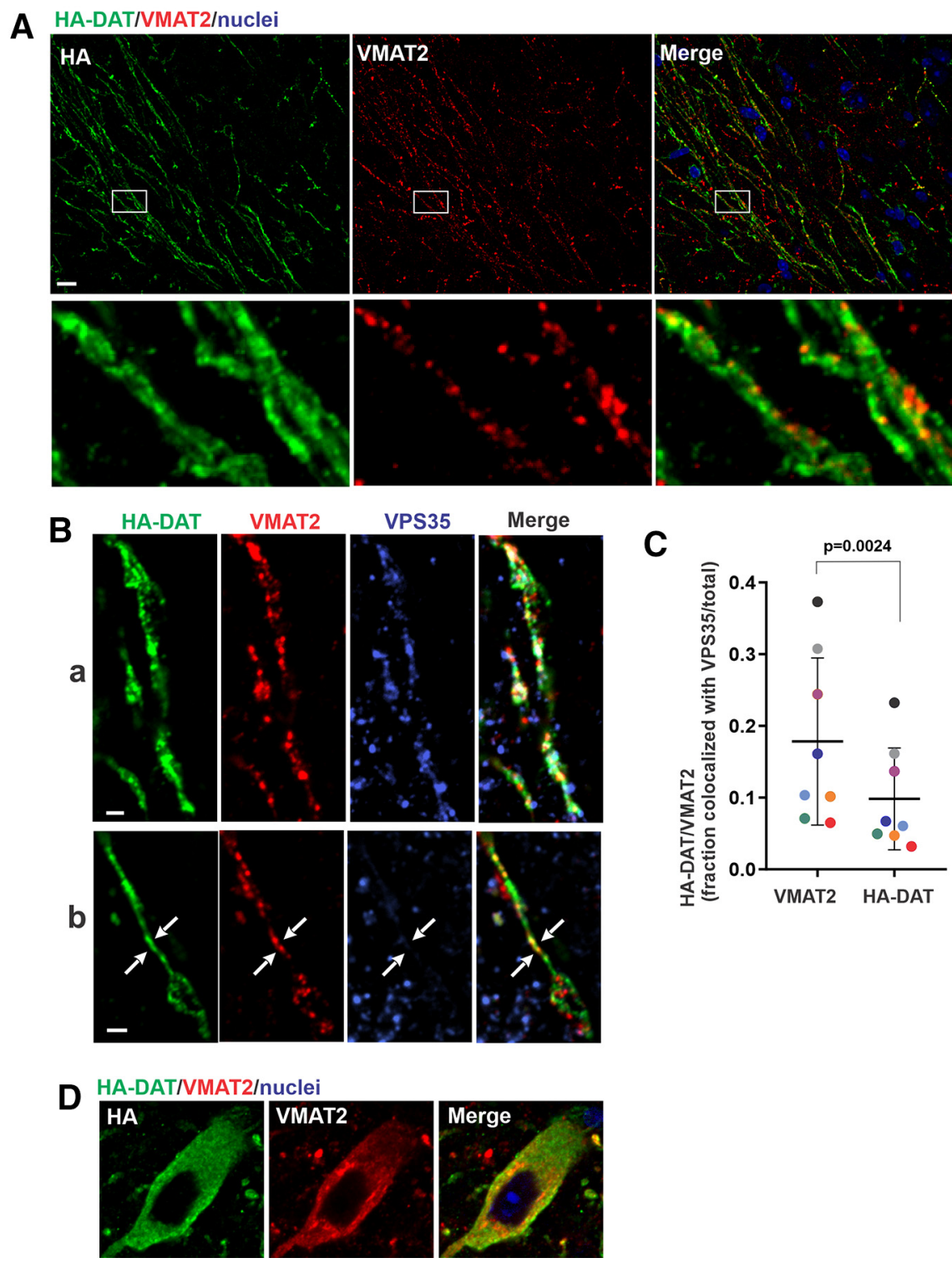

Figure 5. Coimmunolabeling of HA-DAT, VMAT2, and VPS35 in MFB and midbrain. Brains were fixed by cardiac perfusion with PFA and prepared for sectioning as described in Materials and Methods. Sagittal cryosections were colabeled with mouse HA11 and antibodies against VMAT2 $(\boldsymbol{A}, \boldsymbol{D})$, or with mouse HA11, VMAT2, and goat polyclonal VPS35 antibodies $(\boldsymbol{B}, \boldsymbol{C})$ followed by fluorophore-conjugated secondary antibodies. $\boldsymbol{A}, \boldsymbol{D}, 3 \mathrm{D}$ stacks of images were acquired in MFB $(\boldsymbol{A})$ and midbrain areas (D) of cryosections through 640 (red, VMAT2), $488 \mathrm{~nm}$ (green, HA-DAT), and $405 \mathrm{~nm}$ (blue, Hoechst) channels. Maximum intensity projections of three consecutive $x-y$ confocal sections are shown. $\boldsymbol{A}$, Insets, High-magnification images of the region indicated by white rectangles. $\boldsymbol{D}$, Dopaminergic neuron soma. Scale bars, $10 \mu \mathrm{m}$. $\boldsymbol{B}, \boldsymbol{C}, 3 \mathrm{D}$ stacks of images were acquired in MFB through $488 \mathrm{~nm}$ (green, HA-DAT), $561 \mathrm{~nm}$ (red, VMAT2), and $640 \mathrm{~nm}$ (cyan, VPS35) channels. Representative images (single confocal sections) with examples of individual axons containing ( $\boldsymbol{a}$ images) or not containing VPS35 puncta ( $\boldsymbol{b}$ images). Two arrows indicate axon. Scale bars, $2 \mu \mathrm{m}$. Quantification of the fractions of HA-DAT and VMAT2 colocalized with VPS35 in dopaminergic axons was performed as described in Materials and Methods. Data points corresponding to values of fractions of VMAT2 and HA-DAT colocalized with VPS35 quantified in the same images are shown in the same colors. Scatter dot plot represents mean values with SDs. $p$ value was calculated using paired Student's $t$ test ( $n$ of 8 images containing multiple axons).

from an HA-DAT fraction colocalized with nonspecific IgG ( $\sim 1 \%-3 \%$; Fig. $1 G)$, confirming an absence of detectable quantities of specifically labeled late endosomes and lysosomes in MFB dopaminergic axons (Fig. 1G).

By contrast, coimmunolabeling of MFB cryosections with HA11 and antibodies to VPS35, a component of the retromer complex involved in the recycling of cargo from endosomes to the plasma membrane or Golgi complex (Burd and Cullen, 2014), revealed significant colocalization of HA-DAT and VPS35 in the puncta along axonal tracts (Fig. $3 A, B$ ). This result is consistent with a recent report proposing that VPS35 is involved in DAT trafficking in NAc (S. Wu et al., 2017). Colocalization of HA-DAT with VPS35 was especially apparent in the axonal region caudal to striatum (Fig. $3 B$ ). The mean fraction of HA-DAT colocalized with VPS35 was $\sim 7 \%$ (Fig. $1 G$ ). Interestingly, axons were heterogeneous in the abundance of retromer compartments: some contained a lineup of multiple VPS35-labeled puncta, whereas others contained only a single or no detectable VPS35-labeled vesicles. The substantial variability in the fraction of HA-DAT colocalized with VPS35 is also evident in Figure $1 G$. VPS35 endosomes were abundant in the soma (Fig. 3C), suggesting that retromer may be involved in DAT traffic in all subcompartments of a dopaminergic neuron.

To analyze HA-DAT/VPS35 colocalization at a higher resolution, 3D STED super-resolution fluorescence imaging was performed. STED imaging allowed clear visualization of the plasma membrane in some axons and revealed a highly clustered HA-DAT distribution along the plasma membrane (Fig. 4), consistent with the DAT localization pattern in cultured neurons demonstrated by super-resolution microscopy (RahbekClemmensen et al., 2017). A small fraction of HA-DAT puncta were colabeled with the VPS35 antibody (Fig. 4), thus further confirming the localization of HA-DAT in recycling endosomal carriers. Together, data in Figures 1-4 demonstrate that a relatively small pool of DAT is located in VPS35-positive, possibly recycling endosomes, whereas early, sorting, and late endosomes as well as lysosomes are abundant in neuronal soma but appear not to penetrate into MFB axons to a significant extent.

\section{Comparison of localization patterns of DAT and VMAT2 in MFB axons}

To compare HA-DAT localization in MFB axons with that of another transmembrane protein enriched in dopaminergic neurons, we analyzed localization of the VMAT2. VMAT2 was found to be highly colocalized with HA-DAT-positive MFB axons (Fig. 5A), although VMAT2 was also located in nondopaminergic processes, presumably, serotonergic axons. Quantifications revealed that, on average, 37\% of HA-DAT fluorescence $(15 \% \mathrm{SD} ; n=10)$ was colocalized with VMAT2. Importantly, the localization pattern of VMAT2 in MFB axons was predominantly punctate compared with a less-punctate, mostly uninterrupted immunolabeling of HA-DAT along these axons (Fig. $5 A$, insets). To examine whether the punctate 

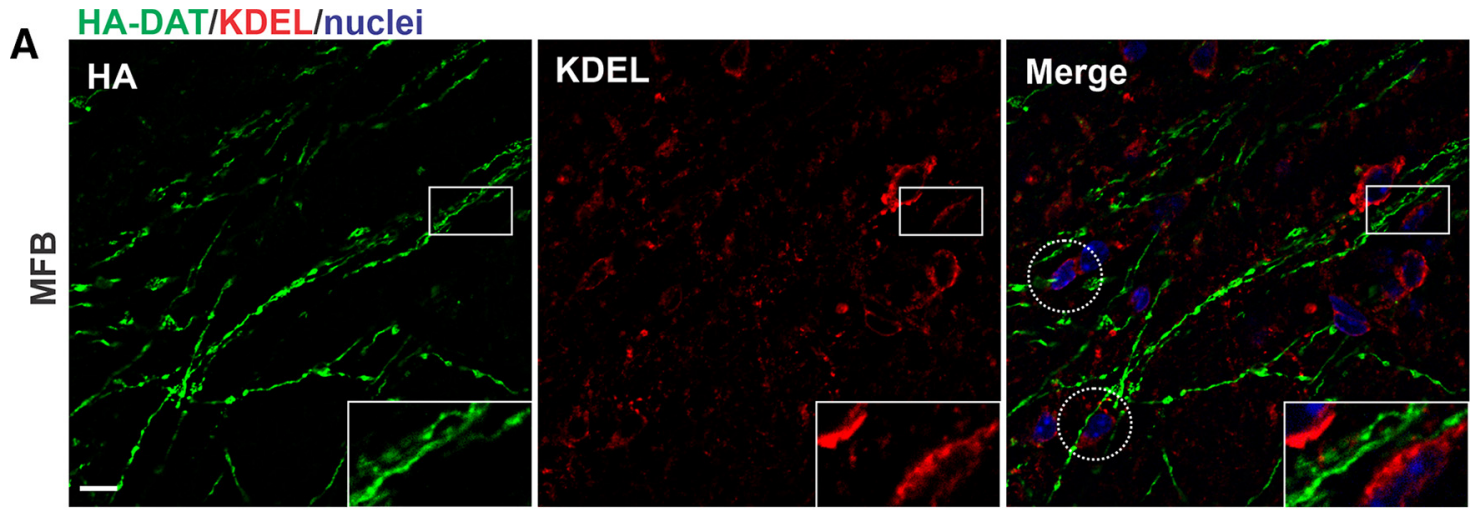

B HA-DAT/KDEL
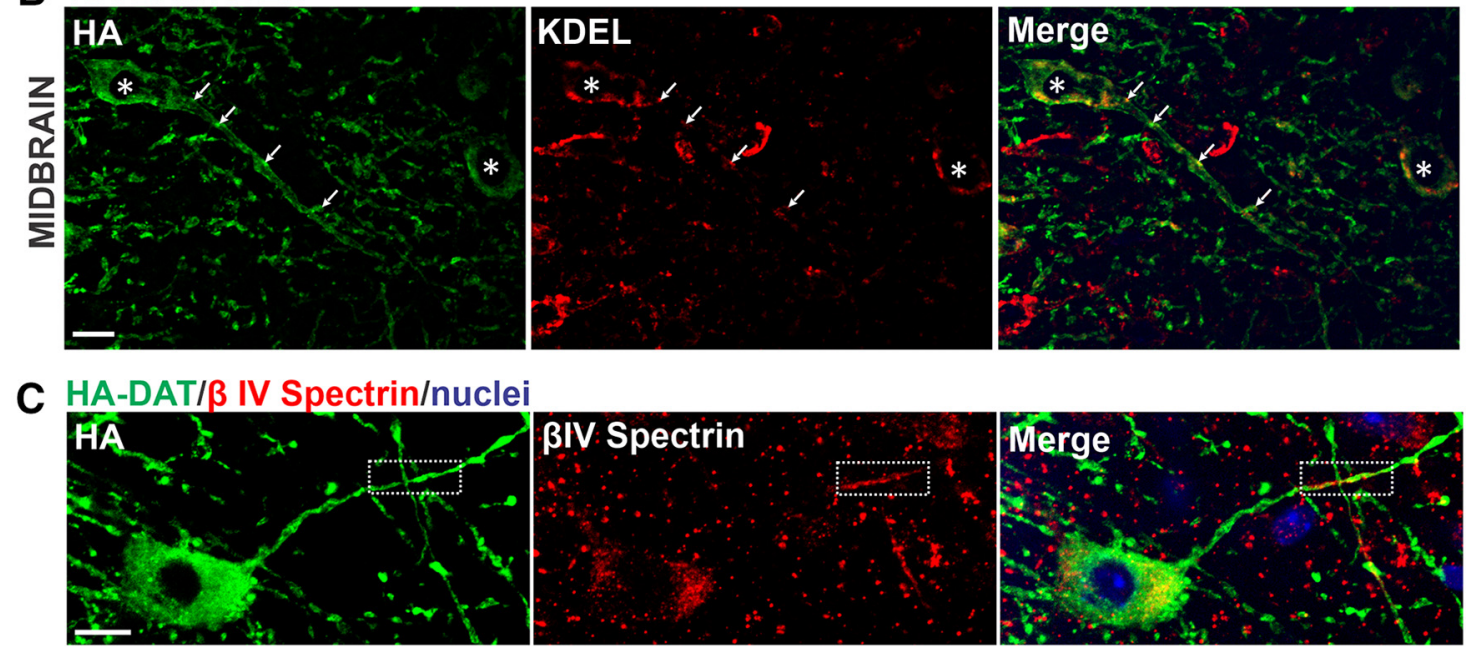

D

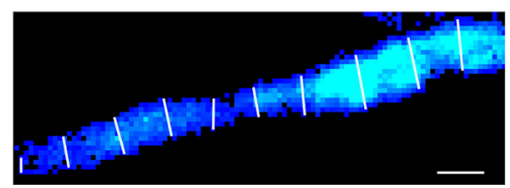

E Parameters (10-20 neurons)

Diameter of axon (MFB) $(\mu \mathrm{m})$ AIS length $(\mu \mathrm{m})$ AIS diameter $(\mu \mathrm{m})$

Soma to proximal end of AIS ( $\mu \mathrm{m})+33.35+3.78 \quad 15.99-49$.

Figure 6. Coimmunolabeling of HA-DAT with markers of ER and AIS in MFB axons and midbrain. Brains were fixed by cardiac perfusion with PFA and prepared for sectioning as described in Materials and Methods. Sagittal cryosections were labeled with HA11 and KDEL $(\boldsymbol{A}, \boldsymbol{B})$ or $\beta \mathrm{IV}$-spectrin $(\boldsymbol{C}, \boldsymbol{D})$ antibodies followed by fluorophore-conjugated secondary antibodies. 3D stacks of $x-y$ confocal images of MFB $(\boldsymbol{A})$ and midbrain $(\boldsymbol{B}-\boldsymbol{D})$ were acquired through $640 \mathrm{~nm}$ (red, KDEL or $\beta$ IV-spectrin), $488 \mathrm{~nm}$ (green, HA-DAT), and $405 \mathrm{~nm}$ (blue, Hoechst) channels. Maximum intensity projections of three consecutive $x-y$ confocal sections are shown. $\boldsymbol{A}$, White circles represent examples of characteristic perinuclear KDEL labeling in HA-DAT-negative (nondopaminergic) cells. Insets represent high-magnification images of the region indicated by white rectangles. $\boldsymbol{B}$, Asterisks indicate soma of dopaminergic neurons. Arrows indicate examples of HA-DAT and KDEL colocalization. C, White rectangles represent the position of the AIS. Multiple puncta of $\beta$ IV-spectrin in the surrounding neuropil likely represent nodes of Ranvier in nondopaminergic myelinated neurons. $\boldsymbol{D}$, High magnification of the area indicated by white rectangles in $\boldsymbol{C}$ represents an example of AIS diameter measurements (indicated by white lines) using image segmentation. Scale bars, $10 \mu \mathrm{m}$. $E$, Measurements of an apparent diameter of dopaminergic axons in the AIS and MFB regions distal to AIS, AIS length, and the distance of the soma-proximal edge of AIS from the soma. Data are mean $\pm S D ; n=10-20$ axons.

pattern of VMAT2 localization in axons is because of its localization in endosomes, triple immunolabeling of VMAT2, HADAT, and VPS35 was performed, and the fractions of two transporters colocalized with VPS35 in dopaminergic axons were quantitated and compared in the same images. Representative examples of individual axons shown in Figure $5 B$ demonstrate heterogeneity in the abundance of VPS35 endosomes in different HA-DAT/VMAT2-positive axons and variability in the extent of VPS35 colocalization with two transporters in individual MFB axons. Importantly, the fraction of VMAT-2 colocalized with VPS35 was higher than that of HA-DAT with VPS35 in each image analyzed, and overall, significantly higher for VMAT2/ VPS35 than HA-DAT/VPS35 colocalization (Fig. 5C). Interestingly, while both HA-DAT and VMAT2 are located in vesicles and ER network throughout the soma, VMAT2 was more concentrated in the perinuclear area, suggesting that sorting of these two transporters begins in the soma (Fig. 5D). Unlike DAT, VMAT2 has a strong di-leucine internalization signal (Tan et al., 1998), and therefore, can be efficiently internalized and accumulated in endosomes. Therefore, the data in Figure 5 illustrate an example of a transmembrane cargo that, in contrast to DAT, may largely rely on vesicular transport mechanisms for long-distance trafficking.

\section{ER-localized DAT is confined to the soma with traces detected in MFB axons}

The prevalent pattern of continuous, uninterrupted fluorescence of HA-DAT in MFB axons may correspond to HA-DAT localization in the plasma membrane or tubular ER. Immunolabeling with antibodies that detect resident ER proteins containing the ER-targeting KDEL sequence motif in their luminal domains demonstrated that these ER proteins were not observed in HADAT-positive axons in MFB (Fig. 6A,B). By contrast, KDEL 


\section{A HA-DAT/RTN3/nuclei}
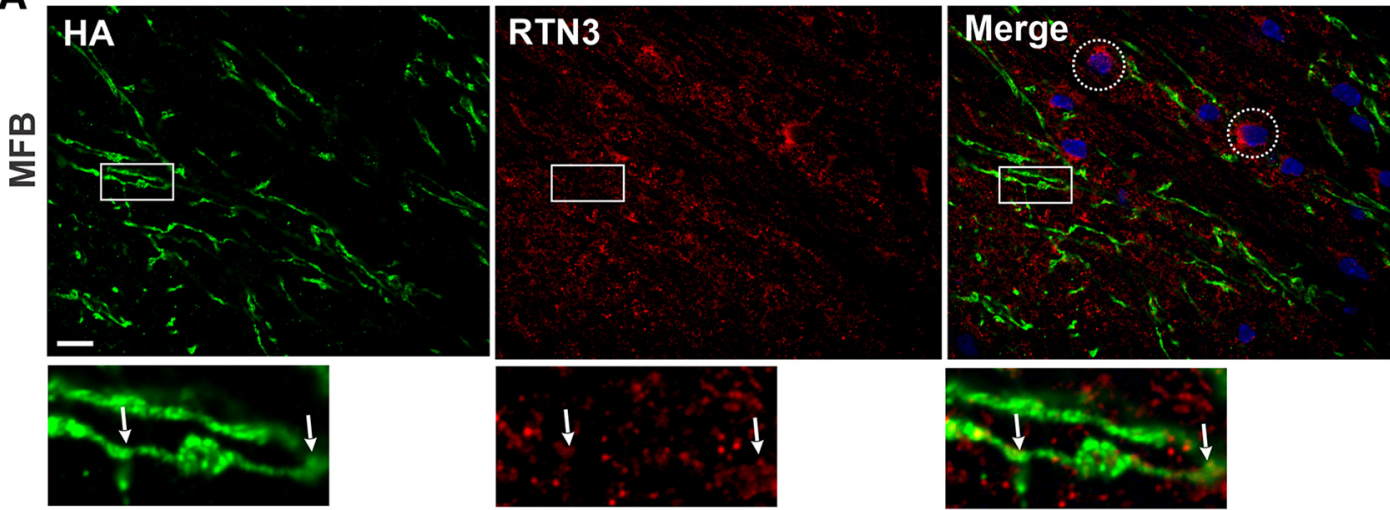

B HA-DAT/RTN3/nuclei
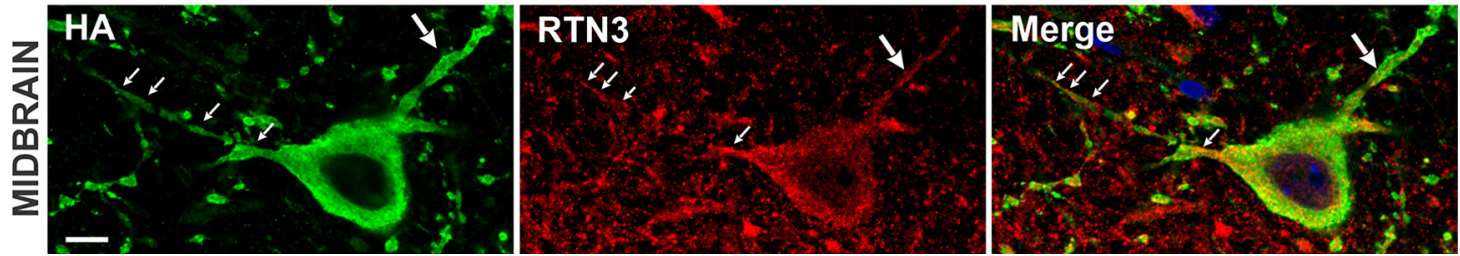

Figure 7. Coimmunolabeling of HA-DAT and RTN3 in MFB axons and midbrain. Brains were fixed by cardiac perfusion with PFA and prepared for sectioning as described in Materials and Methods. Sagittal cryosections were labeled with HA11 and RTN3 antibodies followed by fluorophore-conjugated secondary antibodies. 3D stacks of $x-y$ confocal images of MFB ( $\boldsymbol{A}$ ) and midbrain (B) were acquired through $640 \mathrm{~nm}$ (red, RTN3), $488 \mathrm{~nm}$ (green, HA-DAT), and $405 \mathrm{~nm}$ (blue, Hoechst) channels. Maximum intensity projections of three consecutive $x-y$ confocal sections are shown. $\boldsymbol{A}$, White circles represent examples of characteristic perinuclear RNT3 labeling in HA-DAT-negative (nondopaminergic) cells. Insets, High-magnification images of the area indicated by white rectangles in $\boldsymbol{A}$. $\boldsymbol{A}$, Small arrows indicate examples of traces of RTN3 fluorescence colocalized with HA-DAT in distal MFB axons. $\boldsymbol{B}$, Small arrows indicate RTN3 fluorescence in somaproximal axons positive for HA-DAT. Large arrow indicates a dendrite. Scale bars, $10 \mu \mathrm{m}$.

proteins could be readily detected in nondopaminergic cells in MFB (Fig. 6A) and the soma of dopaminergic neurons where KDEL staining strongly overlapped with HA-DAT (Fig. 6B). In some of the latter cells, KDEL staining extended $20-40 \mu \mathrm{m}$ from the soma into the proximal part of the axon, where KDEL was seen colocalized with HA-DAT (Fig. 6B). Interestingly, AISs in dopaminergic neurons were found to be located at a similar distance from the soma $(30-40 \mu \mathrm{m}$; Fig. $6 C-E)$, suggesting that the AIS may limit or minimize further extension of the ER into the axonal processes. At its origin, the AIS of dopaminergic axons reportedly has a mean diameter of $0.37 \mu \mathrm{m}$, tapering by half over the AIS length (González-Cabrera et al., 2017). Our measurements from confocal images, however, yielded somewhat larger dimensions (mean of $0.65 \mu \mathrm{m}$ ), which were comparable to the size of MFB axons (mean of $0.5 \mu \mathrm{m}$ ) and did not demonstrate narrowing of the axon at the AIS (Fig. $6 C-E$ ).

Similarly to KDEL proteins, RTN3, a resident ER protein, including the smooth ER, was largely undetectable in MFB axons (Fig. $7 A$ ), but enriched in the perinuclear area of dopaminergic and other cells (Fig. 7A,B). Infrequently, traces of RTN3 were detected in HA11-labeled axons (Fig. 7A, insets), although the extent of HA-DAT/RTN3 colocalization was technically difficult to quantify because of an extensive RTN3 labeling in cells surrounding HA-DAT-labeled axons and thus low signal-to-noise ratio. Notably, RTN3 labeling extended from the soma into dendrites and proximal axons in dopaminergic neurons (Fig. $7 B$ ). It is important to consider that, even if the ER labeled by these two markers in MFB axons is below the detection limit of the fluorescence imaging, considering the ratio of HA-DAT and ER marker fluorescence in the soma (Figs. $6 C, 7 B$ ), and assuming that such a ratio is maintained throughout the ER in axons, the amount of HA-DAT in axonal ER is expected to be negligible. Together, the data in Figures 6 and 7 suggest that the HA-DAT distribution pattern in MFB axons is consistent with a predominant localization in the plasma membrane and a minimal presence in the ER.

\section{Immunolabeling of HA-DAT in MFB axons in intact brain} by injecting HA11 antibodies

The data in previous figures ruled out the existence of a significant pool of HA-DAT in endocytic compartments and ER in MFB axons, suggesting that the intensive HA-DAT immunolabeling is mainly associated with the plasma membrane. To directly visualize cell-surface HA-DAT in the intact mouse brain, we performed stereotactic injections of the HA11 antibody into the MFB area (Fig. 8A). As shown in Figure 8B, injected antibodies are expected to bind cell-surface HA-DAT, but not HA-DAT that is associated with intracellular membranes at the time of injection. Subsequent incubation of fixed and detergent-permeabilized sagittal slices with rabbit HA-epitope antibodies $(\mathrm{R} \alpha \mathrm{HA})$ is expected to allow the immunolabeling of intracellular HA-DAT as well as cell-surface HA-DAT that was not occupied by HA11 (Fig. 8B). Figure $8 C$ shows a strong HA11fluorescence (green), corresponding to plasma membrane HA-DAT, and $\mathrm{R} \alpha \mathrm{HA}$ (red), corresponding to total HA-DAT. HA11 and R $\alpha \mathrm{HA}$ labeling patterns were highly similar and strongly overlapped along axons with rarely detectable puncta that was labeled with $\mathrm{R} \alpha \mathrm{HA}$ but not with HA11 (corresponding to intracellular HADAT) (Fig. 8C). Pearson correlation coefficients were high with a mean value of 0.59 , despite that injected HA11 inevitably exhibited nonspecific binding to nondopaminergic cells (Fig. $8 C$ ). Colabeling with the VPS35 antibody demonstrated that only few R $\alpha$ HA-labeled dots (not labeled with HA11) could be defined as endosomes (Fig. 8C, right, insets). These data demonstrate the predominant localization of HA-DAT in the plasma membrane of MFB axons in intact brain. 


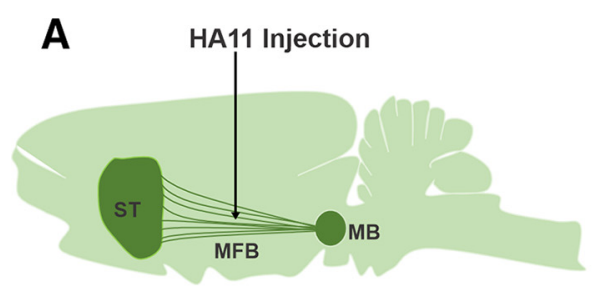

Coordinates

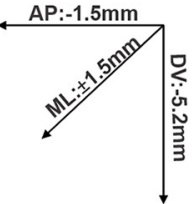

B
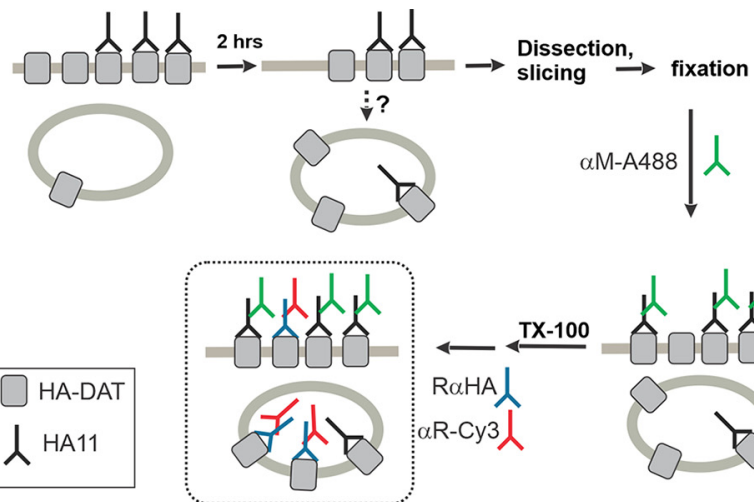

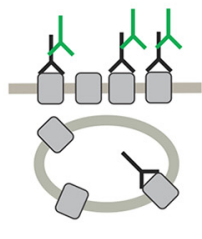

C
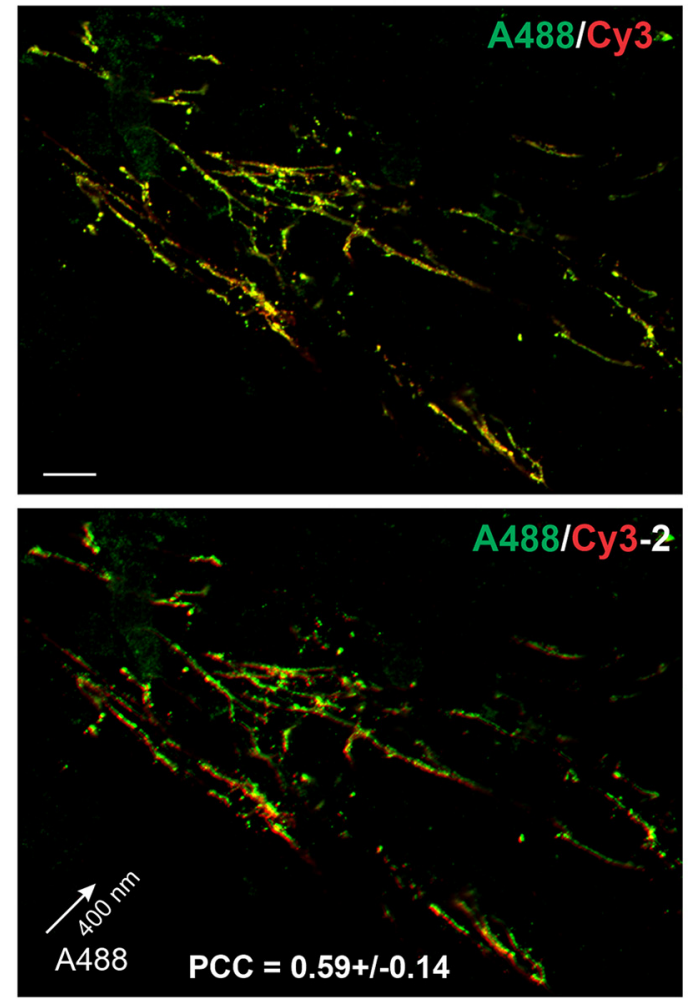
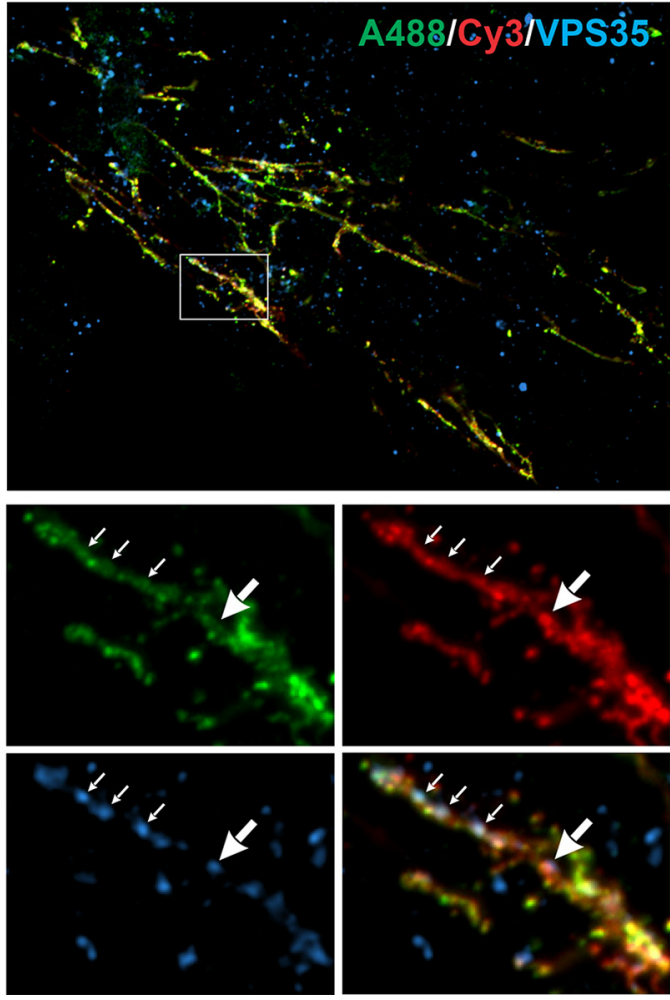

Figure 8. Labeling of HA-DAT in MFB axons by stereotactic injection of HA11 antibodies in vivo. A, Stereotactic injections of HA11 antibodies into the MFB area were performed as described in Materials and Methods. $\boldsymbol{B}$, Schematics of the experimental protocol for labeling plasma membrane and intracellular HA-DAT. Injected antibody binds to a fraction of plasma membrane HADAT and internalizes during animal recovery after injections $(\sim 2 \mathrm{~h}$ ). Animals are then killed, brains are dissected, and sagittal slices are prepared and fixed. Fixed slices are incubated with the secondary A488-conjugated donkey anti-mouse-lgG antibody ( $\alpha \mathrm{M}$-A488) at $4^{\circ} \mathrm{C}$ to label HA11:HA-DAT complexes remaining on the surface of neurons. Following permeabilization with Triton X-100 (TX-100), slices are incubated with rabbit monoclonal anti-HA antibody (R $\alpha H A)$ to label intracellular HA-DAT as well as cell-surface HA-DAT that was not occupied by HA11. In the same incubation, goat polyclonal VPS35 antibody (not shown in the protocol scheme) is included. Secondary donkey anti-rabbit and anti-goat antibodies conjugated with (y3 and (y5 are finally used to detect R $\alpha$ HA and VPS35 antibodies, respectively. C, 3D stacks of $x$-y confocal images were acquired from slices in $35 \mathrm{~mm}$ MatTek dishes through $488 \mathrm{~nm}$ (green, HA11), $561 \mathrm{~nm}$ (red, $\mathrm{R} \alpha \mathrm{HA}$ ), and $640 \mathrm{~nm}$ (cyan, VPS35) channels. Maximum intensity projections of three consecutive $x-y$ confocal sections are shown. In the merge A488/Cy3-2 image, A488 fluorescence (HA11, surface HA-DAT) was shifted 4 voxels relative to Cy3 fluorescence (R $\alpha$ HA, total HA-DAT), as shown by an arrow for better visualization of highly similar patterns of A488 and Cy3 fluorescence. Colocalization of $488 \mathrm{~nm}$ (plasma membrane HA-DAT) and $561 \mathrm{~nm}$ (total HA-DAT) channel fluorescence was also estimated by calculating Pearson correlation coefficient (PCC) using SlideBook6 $(n=6)$. Insets, High-magnification images of the region indicated by white rectangle in the merge image of HA11, R $\alpha$ HA, and VPS35 fluorescence. Small arrows indicate VPS35 puncta in the axon that do not colocalize with HA-DAT puncta. Large arrow indicates an example of HA-DAT (R $\alpha$ HA) localization in VPS35 containing endosome that lacks HA11. Scale bar, $10 \mu \mathrm{m}$.

\section{Quantification of morphologic features and HA-DAT} subcellular distribution in MFB using immunogold-labeling EM

At the ultrastructural level, profiles containing specific immunogold labeling for HA-DAT were localized primarily within the nigrostriatal portion of the MFB (Fig. 9A) and were often surrounded by additional immunoreactive structures. Examination of other portions of the MFB outside this relatively clustered zone revealed considerably fewer profiles with immunolabeling. The identification of immunogold-bearing structures as axons was based in part on their expression of HA-DAT, as well as their failure to receive synaptic input and their tendency to be surrounded by small-to-medium sized unlabeled axons (Fig. $9 B-D)$. Clusters of vesicles ordinarily used to identify axons were 

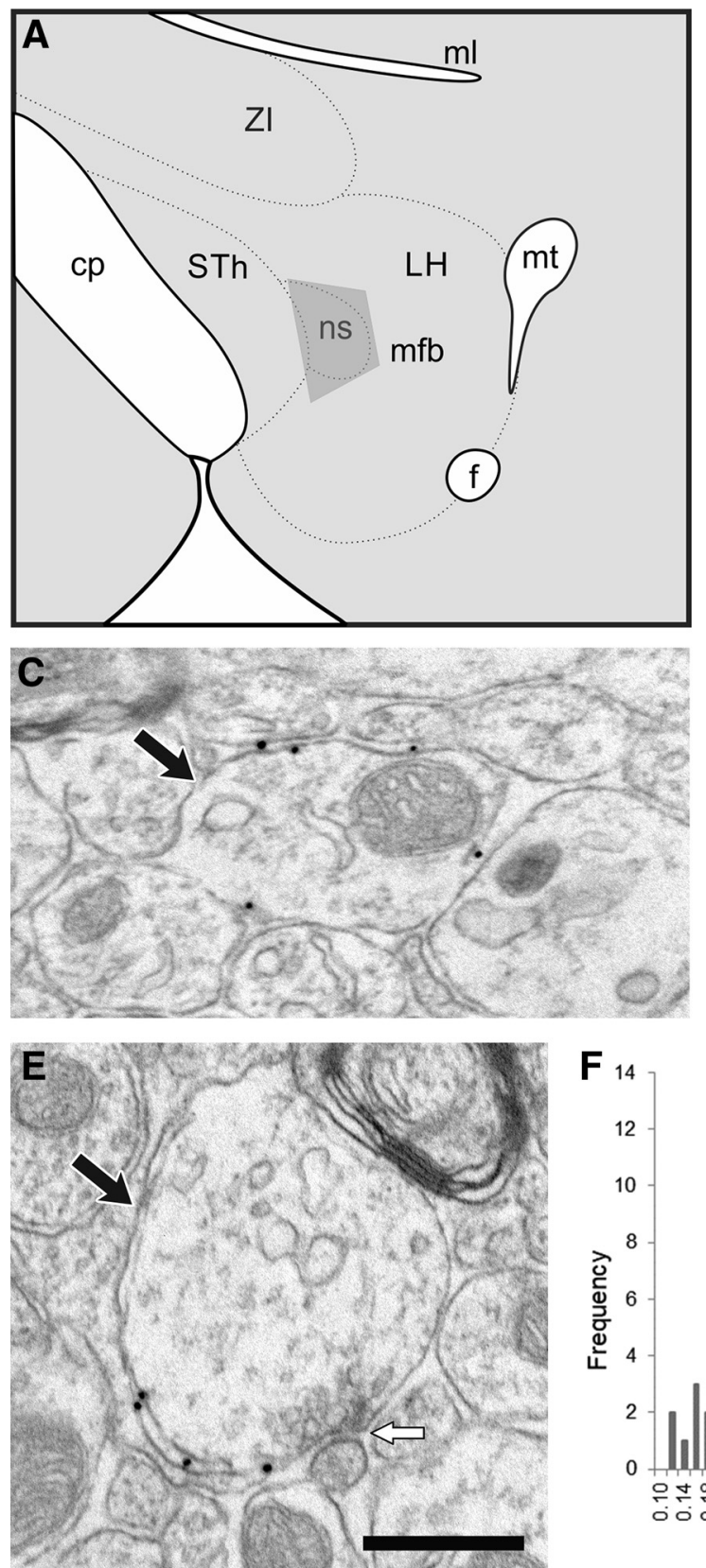

Figure 9. Images illustrating the region sampled by EM and representative axonal profiles varying in size and expressing primarily plasmalemmal immunogold-silver labeling for HA-DAT in the mouse. $A$, The approximate region of the MFB examined for nigrostriatal axons (ns; gray trapezoid represents sectioning by ultramicrotomy) lies medial to the cerebral peduncle (cp) and subthalamic nucleus (STh). Modified from Paxinos and Watson (1998) to resemble mouse brain. f, Fornix; LH, lateral hypothalamus; ml, medial lemniscus; mt, mammillothalamic tract; Zl, zona incerta. $\boldsymbol{B}-\boldsymbol{E}$, Axons (indicated by large black arrows) of increasing short-axis diameter $(0.31,0.48,0.72$, and $0.78 \mu \mathrm{m}$, respectively) show immunogold-silver labeling for HA-DAT exclusively on the plasma membrane and are surrounded by numerous unlabeled axons of comparable or smaller diameter. $\boldsymbol{B}$, Right, Gold particles represent low levels of nonspecific labeling seen in this material. $\boldsymbol{E}$, Small white arrow indicates a cluster of vesicles at an apparent presynaptic specialization. Scale bar: $(\boldsymbol{E}) \boldsymbol{B}-\boldsymbol{E}, 0.4 \mu \mathrm{m}$. $\boldsymbol{F}$, Frequency histogram of diameter measurements from 210 axons labeled for HA-DAT from the nigrostriatal portion of the MFB of 4 HA-DAT knock-in mice. The data indicate considerable variability in the short-axis diameter measured in the electron microscope from single sections.

seen rarely in labeled profiles, typically at sites with presynapticlike features (Fig. 9E). Otherwise, round $\sim 40-50 \mathrm{~nm}$ vesicular (circular) profiles were observed individually within profiles labeled for HA-DAT, which also contained somewhat larger, endosome-like organelles and mitochondria (Fig. 9).

From the four HA-DAT mice examined, 210 immunogold-labeled profiles (47-60 per animal) with these morphologic features were assessed for their size and for the subcellular localization of HA-DAT. The short-axis diameter of HA-DATlabeled profiles ranged from 0.12 to $1.24 \mu \mathrm{m}$ (Fig. $9 E$ ), with a
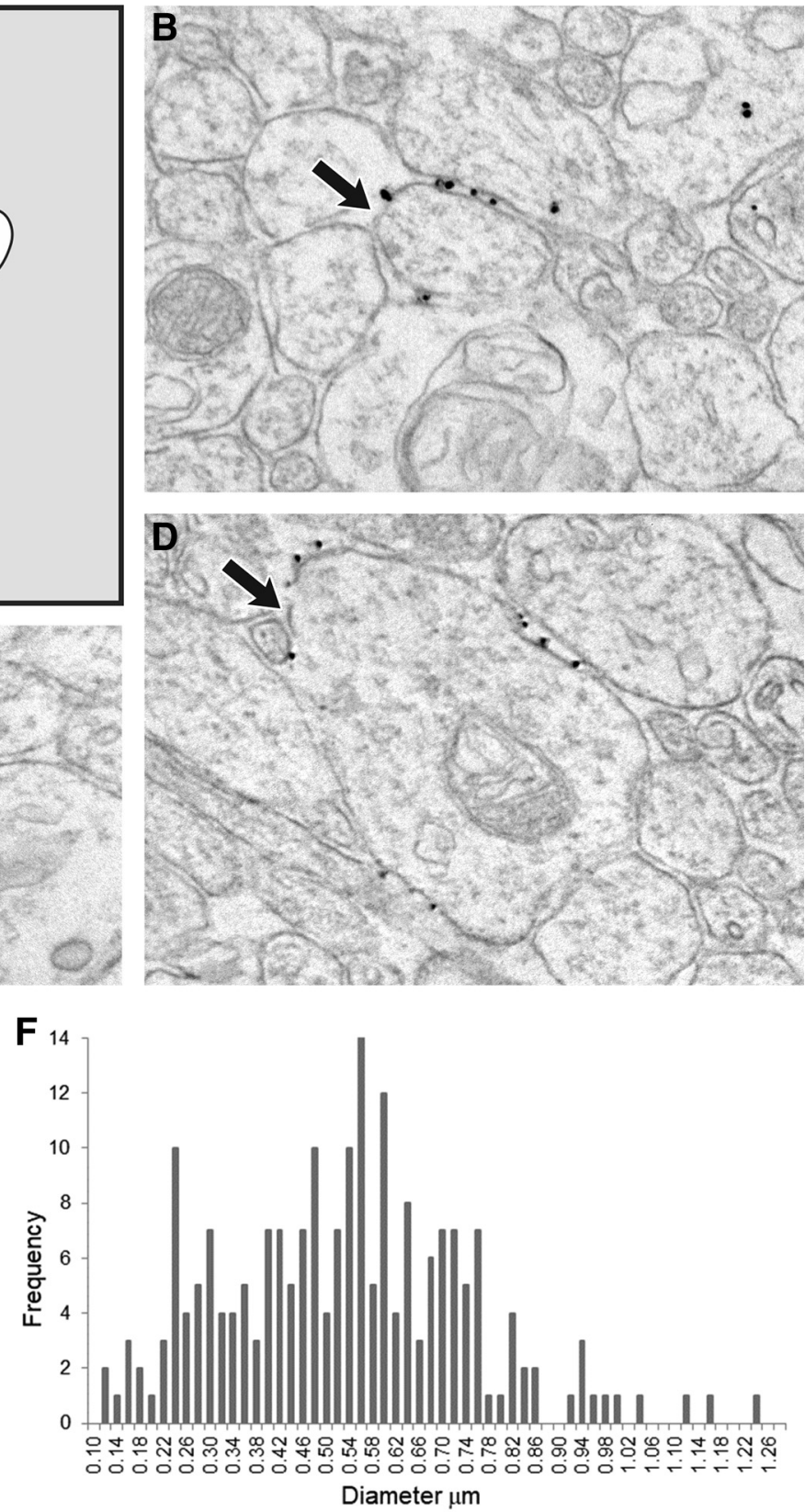

Diameter $\mu \mathrm{m}$ 
Table 1. Size of axons labeled for HA-DAT in the mouse nigrostriatal pathway

\begin{tabular}{lllll}
\hline Animal & No. of axons & Perimeter $(\mu \mathrm{m})$ & Area $\left(\mu \mathrm{m}^{2}\right)$ & Diameter $(\mu \mathrm{m})$ \\
\hline 1 & 47 & 2.304 & 0.349 & 0.514 \\
2 & 48 & 2.462 & 0.397 & 0.431 \\
3 & 60 & 2.342 & 0.380 & 0.585 \\
4 & 55 & 2.197 & 0.344 & 0.560 \\
$N$ & 210 & & & \\
Mean & & 2.326 & 0.368 & 0.522 \\
SEM & & 0.055 & 0.013 & 0.034 \\
CV & & $2 \%$ & $3 \%$ & $6 \%$ \\
\hline
\end{tabular}

Quantitative data showing the size of axons labeled by immunogold-silver for HA-DAT in 4 knock-in mice. Size is estimated by membrane perimeter, cross-sectional area, and short-axis diameter (see Materials and Methods). Coefficients of variation $(\mathrm{CV})<10 \%$ indicate relatively low variability in these measures across animals.

in fluorescence intensities. A similar width range of MFB axons was estimated from measurements in STED images of HA-DAT (Fig. 4).

The majority of immunogold-silver particles for HA-DAT were localized to the plasma membrane of axons within the nigrostriatal MFB (Figs. 9, 10) similar to axon varicosities in the striatum (Block et al., 2015). The exact fraction of plasma membrane gold, however, appeared to be lower within the MFB ( 68\%; Fig. 10) than the dorsolateral striatum (85\%) (see Block et al., 2015). In addition to plasmalemmal labeling for HA-DAT, gold particles were also observed intracellularly (Figs. 9, 10), in some cases in close association with small membrane compartments. The extent to which gold particles were associated with the plasma membrane versus intracellular membranes or the cytoplasm was significant by ANOVA with Tukey's multiple comparison $(p<0.001$; Fig. $10 H)$. For the intracellular organelles associated with HA-DAT gold, a few of these were the approximate size of synaptic vesicles (Fig. $10 A, E$ ), whereas others had slightly larger dimensions and appeared to be small endosomes (Fig. $10 A-F)$. Occasionally, the latter compartments contained multiple immunogold particles for HA-DAT (Fig. 10C,D,F). Based on the mean area of immunolabeled organelles and assuming circularity, the average diameter of intracellular structures was estimated at $\sim 66 \mathrm{~nm}$. Finally, HA-DAT immunogold labeling was infrequently observed within multivesicular bodies (Fig. 10G). In fields intermixed with or immediately adjacent to those sampled for specific labeling, 173 profiles (31-51 per animal) with similar morphologic appearance to those labeled for HA-DAT and comparable to them in area and perimeter ( $p \geq 0.29$, unpaired Student's $t$ test assuming unequal variance) were assessed for nonspecific labeling. This was estimated as $10.2 \%$ for gold particles associated with the plasma membrane, $19.7 \%$ for particles associated with intracellular membranes, and $21.2 \%$ for gold particles in the cytoplasm. These estimates were used to adjust the data for specific labeling before graphing and statistical analysis (Fig. 10H).

\section{FRAP analysis of DAT mobility in living brain slices}

Fluorescence and EM analysis of MFB axons demonstrated that most HA-DAT molecules were located in the plasma membrane, suggesting a major contribution of lateral diffusion in the longdistance traffic of DAT between somatodendritic and axonal compartments of dopaminergic neurons. To measure the diffusion rates of HA-DAT in axonal membranes, HA-DAT was immunolabeled with HA.11 antibody that was complexed with the Fab fragment of secondary anti-mouse-IgG A488-conjugated antibody, in living acute sagittal brain slices. These slices have been characterized in our previous study (Block et al., 2015). Figure $11 \mathrm{~A}$ shows examples of live-cell images of MFB axonal tracts. The localization pattern of HA-DAT:antibody complexes was similar to that of HA-DAT on fixed cryosections, with continuous HA-DAT fluorescence along axons, occasional axonal varicosities, and HA-DAT clusters. In agreement with measurements in fixed neurons (Fig. 6E), MFB axons on live slices were apparently thicker ( $\sim$ twofold) than axonal segments between varicosities in the striatum (Fig. 11A). The FRAP method was used to measure the parameters of HA-DAT diffusion (recovery half-time $\tau_{\mathrm{D}}$, and the $M f$ ) in MFB axonal areas and compare these parameters with those obtained in intervaricose axonal segments in the dStr. As shown in examples of FRAP imaging (Fig. 11B), an $\sim 6$ $\mu \mathrm{m}$ length of axon was photobleached and then monitored for fluorescence recovery. Given the cylindrical shape of axons, a 1D diffusion model was applied to calculate diffusion coefficients $D$ using $\tau_{\mathrm{D}}$ values as described in Materials and Methods. The mean value of $D$ was found to be $\sim 0.55 \mu^{2} / \mathrm{s}$ in MFB and $0.89 \mu^{2} / \mathrm{s}$ in $\mathrm{d}$ Str, suggesting a slightly faster diffusion in striatal axons (Fig. $11 C$ ). Interestingly, on average, only $\sim 35 \%$ of HA-DAT fluorescence was recovered in both MFB and striatal axons (Fig. 11D), indicative of a substantial immobile pool of HA-DAT.

The fact that the fluorescence recovery data were fit using the analytic solution for a diffusion process suggests a minimal contribution of the vesicular traffic in these measurements. Indeed, vesicular-type rapid movement of HA-DAT puncta was not observed in the time frame of FRAP experiments ( 5 min timelapse imaging). Furthermore, to rule out the contribution of endocytic trafficking in FRAP measurements, they were performed at room temperature (typically $22^{\circ} \mathrm{C}$ ), conditions dramatically impairing endocytosis, including endocytosis of DAT (Wiley and Cunningham, 1982; Block et al., 2015; Sorkina et al., 2018). Figure $11 C$ shows that mean values of $D$ at room temperature were slightly lower $\left(0.41 \mu^{2} / \mathrm{s}\right.$ in MFB and $0.84 \mu^{2} / \mathrm{s}$ in dStr) than these values at $37^{\circ} \mathrm{C}$, which agrees with prior reports of weak temperature dependence of the mobility of integral membrane proteins (Hillman and Schlessinger, 1982).

Finally, to directly demonstrate the predominantly plasma membrane localization of HA-DAT under conditions of FRAP experiments at $37^{\circ} \mathrm{C}$, acute slices were incubated with the HA11 antibody and the Cy3-labeled Fab fragment of the secondary antibody recognizing specifically the Fc $\gamma$ domain of mouse IgG at $37^{\circ} \mathrm{C}$. This procedure enables labeling of HA-DAT located at the cell surface and capable of endocytosis (see experimental scheme in Fig. 11E). HA11:HA-DAT complexes remaining at the cell surface were then labeled with A488-conjugated Fab fragments of the secondary antibody recognizing the entire mouse IgG at $4^{\circ} \mathrm{C}$. In this approach, Cy3 fluorescence (red) that does not overlap with A488 fluorescence (green) is considered as internalized HA11:HA-DAT complexes. However, Cy3 and A488 fluorescence signals were remarkably well colocalized with the mean value of Pearson correlation coefficient of $0.76 \pm 0.11$ (SD; $n=6)$; structures labeled with Cy3 but not with A488 were rare, suggesting minimal, if any, endocytosis of HA11:HA-DAT complexes or HA11 alone under the conditions of the FRAP measurements (Fig. 11F). Thus, if diffusion is the major mechanism of DAT long-distance transport, values of $D$ presented in Figure 11 predict (using Eq. 2) that $\sim 21 \mathrm{~d}$ would be necessary to turn over $50 \%$ of the plasma membrane DAT in MFB axonal tracks (on average $2 \mathrm{~mm}$ in length), assuming an unlimited source of DAT in mesencephalon and striatum.

\section{Discussion}

We aimed to determine how DAT is transported between midbrain and striatum by examining DAT localization and 

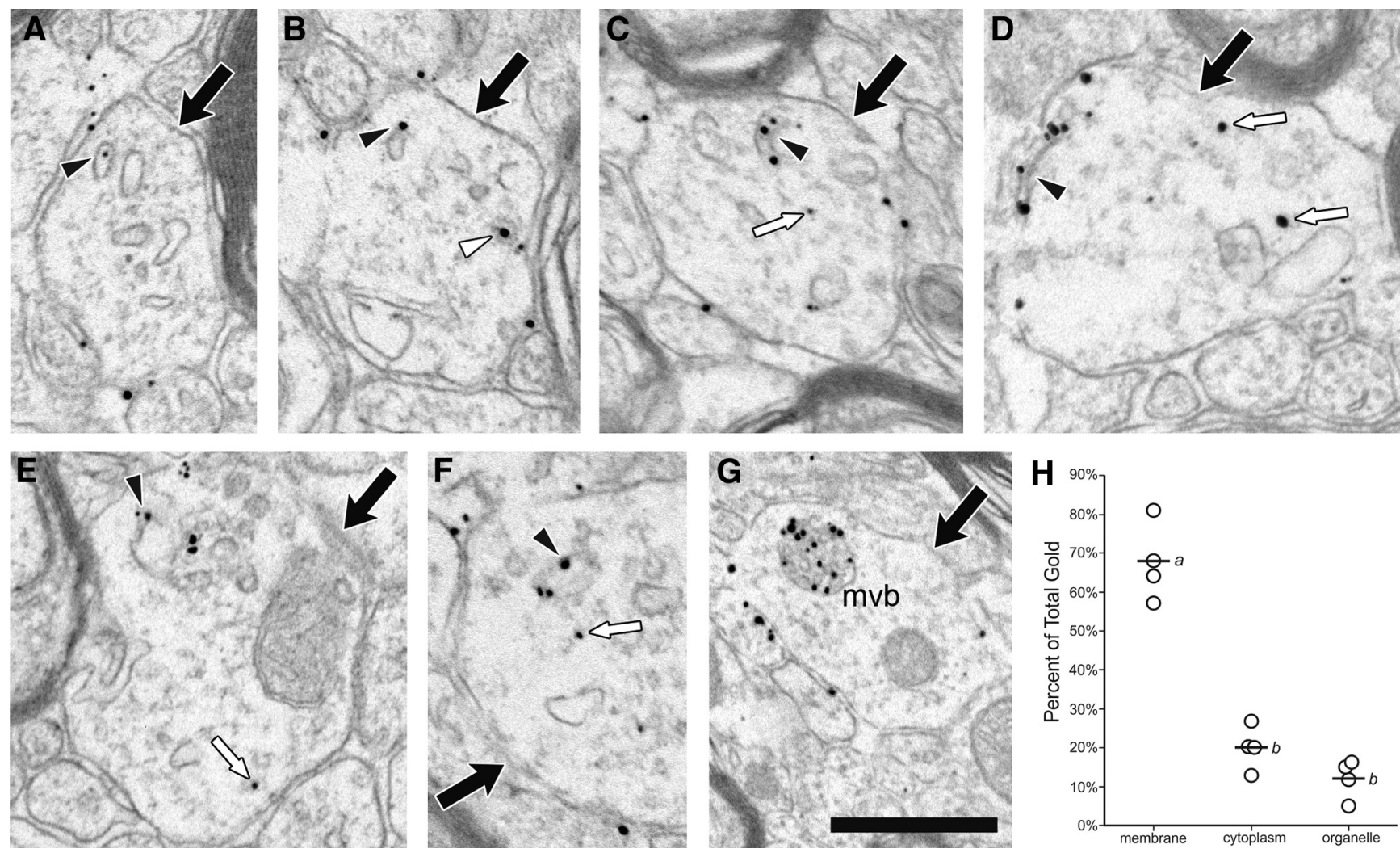

Figure 10. Electron micrographs depicting axons from the MFB (large black arrows) and the subcellular localization of intracellular gold particles representing HA-DAT. $\boldsymbol{A}, \boldsymbol{B}$, In addition to immunoreactivity found along the plasmalemma, gold particles for HA-DAT are sometimes observed in association with vesicles (white arrowheads) or small endosomes (black arrowheads) and are sometimes visible inside these structures. $\boldsymbol{C}, \boldsymbol{D}, 0$ ccasionally, multiple gold particles for HA-DAT are found within single endosomes. $\boldsymbol{E}, \boldsymbol{F}$, Less commonly, HA-DAT labeling is observed in or near slightly larger endosomes. $(-\boldsymbol{F}$, Intracellular gold particles often lack visible contact with membranous structures in single planes of section (small white arrows). $\mathbf{G}$, Immunogold-silver particles for HA-DAT are infrequently observed in association with multivesicular bodies (mvb). Scale bars: (in $\boldsymbol{G}) \boldsymbol{A}-\boldsymbol{G}, 0.4 \mu \mathrm{m}$. $\boldsymbol{H}$, Subcellular distribution of gold particles for HA-DAT in MFB axons for each of 4 mice (open circles), corrected for nonspecific labeling and shown as a percentage of the total gold particles. Solid lines indicate mean values across animals; those with different letters are significantly different $(p<0.0001)$.

membrane mobility in the MFB using confocal, super-resolution fluorescence, and EM. Observations from this analysis are compared with published data on DAT localization in dendritic and striatal axonal compartments of dopaminergic neurons (Nirenberg et al., 1996, 1997; Block et al., 2015). Overall, our studies demonstrate that the pattern of DAT localization in the MFB is somewhat intermediate or "transitional" between somatodendritic and striatal axonal compartments, i.e. the extensive plasma membrane component resembles the striatum, whereas the intracellular DAT pool in MFB axons is smaller than in soma but slightly larger than in striatal axons. We therefore propose that bidirectional DAT trafficking through the MFB involves primarily lateral diffusion in the plasma membrane and, less commonly, vesicular transport.

In characterizing intracellular DAT in MFB axons, VPS35 was the only endosomal protein found significantly colocalized with DAT puncta; and overall, VPS35-labeled endosomes were clearly detectable in some, although not all, dopaminergic axons in MFB. Because microtubule-mediated vesicular transport is at least 10- to 20-fold faster than membrane diffusion, the contribution of the former in DAT traffic through MFB can be significant in the population of axons enriched with VPS35 vesicles. Unfortunately, we have not yet identified adequate antibodies to immunolabel cryosections for sorting nexins, which partner with retromer to determine the specific pathway of retromer-mediated recycling. Therefore, it is unclear whether VPS35-positive compartments participate in anterograde or retrograde DAT transport, local recycling, or all of these. Notably, the VPS35 D620N mutation has been implicated in familial Parkinson's disease, and demonstrated to have pleiotropic effects on the endolysosomal system, autophagy and mitochondrial dynamics in neurons (Rahman and Morrison, 2019). Levels of DAT were reduced, whereas those of VMAT2 were increased in the D620N knock-in mouse (Cataldi et al., 2018), suggesting that VPS35 dysfunction may have a long-term impact on both anterograde and retrograde traffic of these transporters.

A virtual lack of EEA1-positive early/sorting endosomes and CD63/LAMP1-containing late endosomes and lysosomes in MFB was not unexpected, given that we have previously found these compartments to be absent from dStr axons in ex vivo slice preparations and cultured dopaminergic neurons (Rao et al., 2012; Block et al., 2015). Absence of LAMP1 compartments in dopaminergic axons strongly indicates that lysosomal degradation of membrane proteins in these neurons in the intact brain occurs exclusively in soma. Certainly, membrane trafficking systems differ in distinct central and peripheral neurons. For example, LAMP1 compartments have been detected in axons of cultured DRG neurons where they participate in macroautophagy (Maday and Holzbaur, 2016). In another study, LAMP1 compartments in distal DRG axons were proposed to be late endosomes rather than lysosomes because they did not contain cathepsin (Cheng et al., 2018). Transport of late endosomes and autophagosomes from axonal termini to the soma for fusion 
with lysosomes has been shown in DRGs and cultured cortico-hippocampal neurons (Cheng et al., 2015; Kononenko et al., 2017). Thus, whereas the vast majority of observations of sorting/late endosomes in axons are made in studies of cultured neurons often involving overexpression of resident endosomal proteins, future studies in intact brain may determine whether the confinement of these endosomes to the soma is unique for dopaminergic neurons or a more general property of CNS neurons.

The distribution of specific ER markers in dopaminergic neurons has not, to our knowledge, been studied in intact mouse brain. The tubular-cisternae ER network and its "close-proximity" contacts with the plasma membrane and various organelles have been demonstrated in NAc axons by $3 \mathrm{D}$ reconstructions of $\mathrm{EM}$ images, although the type of neurons studied was not specified (Y. Wu et al., 2017). The presence of ER in axons and synaptic areas has also been shown in myelinated neurons in culture and intact mouse brain using several antibodies, including to KDEL (González et al., 2016), and it has been proposed that axonal ER may enable local lipid and protein synthesis (Jung et al., 2014; Luarte et al., 2018). However, we detected only traces of ER in dopaminergic MFB axons using KDEL and RNT3 antibodies. It is possible that the concentration of KDEL-containing proteins and RTN3 in narrow tubules of smooth ER in axons is below the detection limit of our microscopy system given the signal-to-noise ratio of detection in brain tissue. Membrane profiles that could be smooth ER were observed in the MFB of dopaminergic axons by EM, although importantly, rough ER was not detected. However, even if DAT is present in smooth ER in axons, the amount of this ER-located DAT would be negligible. Furthermore, our previous studies in cultured dopaminergic neurons demonstrated that DAT mutants unable to exit the ER were confined to the soma (Miranda et al., 2004). Finally, immature, ER-resident low-molecular-weight species of DAT protein have been detected in the midbrain but not in the striatum (Rao et al., 2013). Hence, available evidence suggests that, in dopaminergic neurons, the function of ER in the translation and posttranslational modifications of transmembrane proteins like DAT is spatially limited to the soma.

The lack of detectable ER in MFB axons beyond the AIS and the predominant plasma membrane localization of DAT in these axons suggest that DAT must be trafficked from ER to Golgi complex (for completion of $\mathrm{N}$-glycosylation) and to the plasma
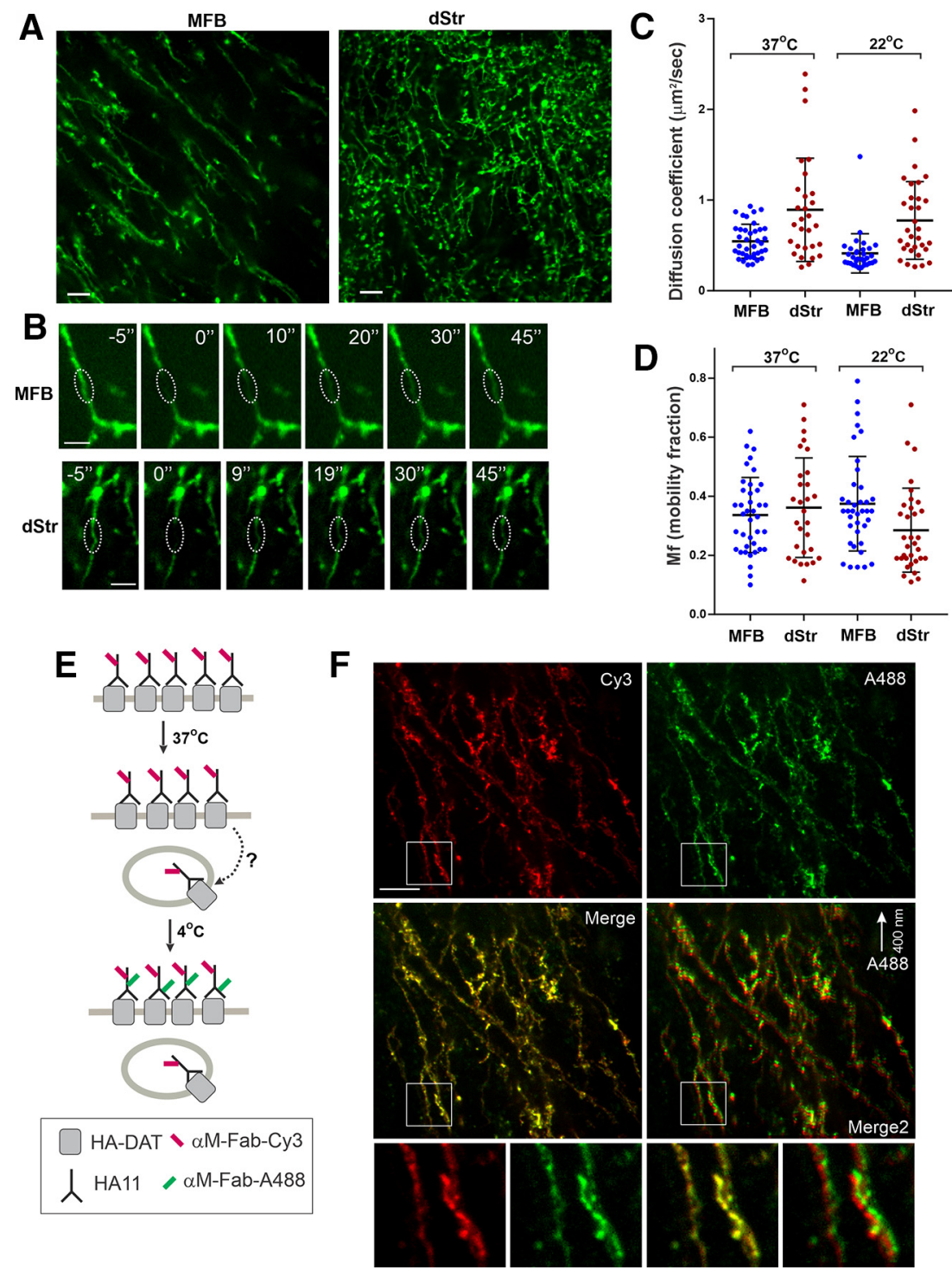

Figure 11. HA-DAT diffusion parameters and internalization in MFB and striatal dopaminergic axons measured in acute sagittal slices. A-D, Acute sagittal brain slices were incubated with the HA11 antibody and Fab fragments of secondary A488-conjugated anti-mouse-lgG antibody at $37^{\circ} \mathrm{C}$ or room temperature $\left(22^{\circ} \mathrm{C}\right)$. Time-lapse images were acquired every $3-5 \mathrm{~s}$ at $37^{\circ} \mathrm{C}$ or $22^{\circ} \mathrm{C}$ before and after bleaching a small region of an axon. $\boldsymbol{A}$, Examples of HA-DAT labeling of MFB and striatum in live slices. Scale bars, $10 \mu \mathrm{m}$. $\boldsymbol{B}$, Selected time frames from the time-lapse sequence before and after bleaching of a region of axons in the MFB and striatal areas. Scale bars, $5 \mu \mathrm{m}$. C, D, Quantification of the diffusion coefficient $(D)$ and $M f$ from three independent series of FRAP experiments exemplified in $\boldsymbol{B}$. Each data point represents calculations of an independent FRAP time-lapse sequence. Differences between $D$ values in MFB and dStr were significant at both $37^{\circ} \mathrm{C}$ and $22^{\circ} \mathrm{C}$ ( $p=0.012$ and $p=0.008$, respectively), whereas differences between $D$ values measured at $37^{\circ} \mathrm{C}$ versus $22^{\circ} \mathrm{C}$ were not significant. Differences between Mf values were not significant for all variants. One-way ANOVA with Tukey's multiple comparison test was used. $\boldsymbol{E}$, Internalization assay in acute slices performed under condition recapitulating FRAP measurements at $37^{\circ} \mathrm{C}$. Slices were incubated with HA11 antibodies and then Cy3-conjugated Fab fragment of goat anti-mouse $\mathrm{Fc} \gamma$-specific antibodies at room temperature. After $40 \mathrm{~min}$ incubation at $37^{\circ} \mathrm{C}$, slices were incubated in nACSF with $5 \mu \mathrm{g} /$ $\mathrm{ml} \mathrm{A488-conjugated} \mathrm{Fab} \mathrm{fragment} \mathrm{of} \mathrm{goat} \mathrm{anti-mouse-lgG} \mathrm{antibodies} \mathrm{for} 30 \mathrm{~min}$ at $4^{\circ} \mathrm{C}$ to label cell-surface HA11:HA-DAT complexes. Slices were then fixed with 4\% PFA. F, 3D imaging was performed through $561 \mathrm{~nm}$ (red, Cy3; surface plus internalized HA-DAT) and $488 \mathrm{~nm}$ (green, A488; surface HA-DAT) channels. Maximum intensity projection images of $z$ stack of 8 consecutive confocal sections are presented. In the Merge 2 image, A488 fluorescence was shifted 4 voxels relative to the Cy3 fluorescence as shown by an arrow for better visualization of virtually identical patterns of A488 and Cy3 fluorescence. Insets, High-magnification images of regions indicated by white rectangles. Scale, $10 \mu \mathrm{m}$.

membrane within the soma-proximal axonal region. AIS protein networks present active and passive barriers to movements of vesicular and plasma membrane cargo (Huang and Rasband, 2018; Leterrier, 2018). DAT expression level in the plasma membrane of the somatodendritic region is less than in MFB axons (Block 
et al., 2015), but it remains to be determined whether the AIS of dopaminergic neurons is a mechanistic transition point between vesicular and diffusive modes of transport. The paucity of evidence for ER or vesicular pools beyond the AIS implies that DAT is transported mostly by lateral diffusion in the plasma membrane through the length of the MFB. With only a minor fraction of DAT localized to vesicles in MFB axons and capable of using a faster microtubule-dependent transport, both anterograde and retrograde transport of DAT is projected to be slow, which explains an extremely slow turnover of DAT protein in vivo $\left(t_{(1 / 2)}>6 \mathrm{~d}\right)$ (Fleckenstein et al., 1996). The inefficiency of DAT long-distance trafficking is further exaggerated by the small Mf of DAT $(\sim 35 \%)$ as demonstrated in our FRAP experiments. For comparison, the Mf of DAT in cultured non-neuronal and dopaminergic cells was $\sim 60 \%-70 \%$, with the exception of N2A cells where this value was 30\% (Adkins et al., 2007; Eriksen et al., 2009; Rao et al., 2012; Sorkina et al., 2013). Interestingly, a similarly small Mf of DAT was observed in filopodia of non-neuronal cells and cultured neurons (Sorkina et al., 2009; Rao et al., 2012). The large immobile pool of DAT in axons could be because of DAT clustering in cholesterol-dependent microdomains (Rahbek-Clemmensen et al., 2017).

EM analysis demonstrated that dopaminergic axons in the MFB had variable diameters that averaged $0.52 \mu \mathrm{m}$. This measurement agreed with the dimensions estimated from confocal and STED images of fixed neurons, as well as living neurons in acute slices. Striatal dopaminergic axons have also been described as variable in diameter (Groves et al., 1994), although the intervaricose segments are thinner, with a mean diameter of $0.24 \mu \mathrm{m}$ (Pickel et al., 1981; Descarries et al., 1996). The difference in thickness of striatal and MFB dopaminergic axons is especially apparent in images just caudal to the striatum, where fibers traversing the MFB begin transitioning to highly branched striatal axons (Fig. 3B). This MFB-striatum transition may cause an organelle "traffic jam," theorized to contribute to neuronal dysfunction and degeneration (Lin et al., 2019), therefore providing pressure to further minimize vesicular traffic in the striatum.

The considerable size variability of dopaminergic axons in the MFB is consistent with other ultrastructural reports of traversing unmyelinated axons in both PNS and CNS, with internal movement of mitochondria and organelles being described as the primary cause of fusiform widenings (Greenberg et al., 1990; Danks et al., 1994; Shepherd et al., 2002). Having variable diameter could slow action potential conduction velocity to something lower than predicted by the smallest diameter of the axon (Greenberg et al., 1990). Hence, the tendency for dopaminergic axons to maintain a wider girth in the MFB might partly compensate for the loss of speed posed by variable diameter (Goldstein and Rall, 1974; Ritchie, 1982). It is also possible that the overall enlarged diameter of dopaminergic axons in the MFB serves to accommodate a higher concentration of mitochondria needed for meeting the metabolic demands of heavily branched striatal axons (Matsuda et al., 2009; Bolam and Pissadaki, 2012; Pissadaki and Bolam, 2013). Indeed, mitochondrial content in unmyelinated axons increases as a proportion of diameter, up to a plateau at $\sim 0.7 \mu \mathrm{m}$ (Perge et al., 2009). This supposition and other theories about how variable diameter might impact organelle and DAT trafficking along different portions of dopaminergic axons will be a subject of future experimentation.

\section{References}

Adkins EM, Samuvel DJ, Fog JU, Eriksen J, Jayanthi LD, Vaegter CB, Ramamoorthy S, Gether U (2007) Membrane mobility and microdomain association of the dopamine transporter studied with fluorescence correlation spectroscopy and fluorescence recovery after photobleaching. Biochemistry 46:10484-10497.

Blasius TL, Reed N, Slepchenko BM, Verhey KJ (2013) Recycling of kinesin-1 motors by diffusion after transport. PLoS One 8:e76081.

Block ER, Nuttle J, Balcita-Pedicino JJ, Caltagarone J, Watkins SC, Sesack SR, Sorkin A (2015) Brain region-specific trafficking of the dopamine transporter. J Neurosci 35:12845-12858.

Bolam JP, Pissadaki EK (2012) Living on the edge with too many mouths to feed: why dopamine neurons die. Mov Disord 27:1478-1483.

Burd C, Cullen PJ (2014) Retromer: a master conductor of endosome sorting. Cold Spring Harb Perspect Biol 6:a16774.

Cataldi S, Follett J, Fox JD, Tatarnikov I, Kadgien C, Gustavsson EK, Khinda J, Milnerwood AJ, Farrer MJ (2018) Altered dopamine release and monoamine transporters in Vps35 p.D620N knock-in mice. NPJ Parkinsons Dis 4:27.

Chen JW, Pan W, D’Souza MP, August JT (1985) Lysosome-associated membrane proteins: characterization of LAMP-1 of macrophage P388 and mouse embryo 3T3 cultured cells. Arch Biochem Biophys 239:574-586.

Cheng XT, Zhou B, Lin MY, Cai Q, Sheng ZH (2015) Axonal autophagosomes recruit dynein for retrograde transport through fusion with late endosomes. J Cell Biol 209:377-386.

Cheng XT, Xie YX, Zhou B, Huang N, Farfel-Becker T, Sheng ZH (2018) Characterization of LAMP1-labeled nondegradative lysosomal and endocytic compartments in neurons. J Cell Biol 217:3127-3139.

Ciliax BJ, Drash GW, Staley JK, Haber S, Mobley CJ, Miller GW, Mufson EJ, Mash DC, Levey AI (1999) Immunocytochemical localization of the dopamine transporter in human brain. J Comp Neurol 409:38-56.

Colak D, Ji SJ, Porse BT, Jaffrey SR (2013) Regulation of axon guidance by compartmentalized nonsense-mediated mRNA decay. Cell 153:12521265.

Dani JA, Zhou FM (2004) Selective dopamine filter of glutamate striatal afferents. Neuron 42:522-524.

Danks AM, Kim P, Wang Z, Meyer RL (1994) Imaging of individual normal and regenerating optic fibers in the brain of living adult goldfish. J Comp Neurol 345:253-266.

Descarries L, Watkins KC, Garcia S, Bosler O, Doucet G (1996) Dual character, asynaptic and synaptic, of the dopamine innervation in adult rat neostriatum: a quantitative autoradiographic and immunocytochemical analysis. J Comp Neurol 375:167-186.

Eriksen J, Rasmussen SG, Rasmussen TN, Vaegter CB, Cha JH, Zou MF, Newman AH, Gether U (2009) Visualization of dopamine transporter trafficking in live neurons by use of fluorescent cocaine analogs. J Neurosci 29:6794-6808.

Fleckenstein AE, Pögün S, Carroll FI, Kuhar MJ (1996) Recovery of dopamine transporter binding and function after intrastriatal administration of the irreversible inhibitor RTI-76 [ 3 beta-(3p-chlorophenyl) tropan-2 beta-carboxylic acid p-isothiocyanatophenylethyl ester hydrochloride]. J Pharmacol Exp Ther 279:200-206.

Gainetdinov RR, Caron MG (2003) Monoamine transporters: from genes to behavior. Annu Rev Pharmacol Toxicol 43:261-284.

Giros B, Caron MG (1993) Molecular characterization of the dopamine transporter. Trends Pharmacol Sci 14:43-49.

Giros B, El Mestikawy S, Bertrand L, Caron MG (1991) Cloning and functional characterization of a cocaine-sensitive dopamine transporter. FEBS Lett 295:149-154.

Giros B, Jaber M, Jones SR, Wightman RM, Caron MG (1996) Hyperlocomotion and indifference to cocaine and amphetamine in mice lacking the dopamine transporter. Nature 379:606-612.

Goldstein SS, Rall W (1974) Changes of action potential shape and velocity for changing core conductor geometry. Biophys J 14:731-757.

González C, Cánovas J, Fresno J, Couve E, Court FA, Couve A (2016) Axons provide the secretory machinery for trafficking of voltage-gated sodium channels in peripheral nerve. Proc Natl Acad Sci USA 113:1823-1828.

González-Cabrera C, Meza R, Ulloa L, Merino-Sepúlveda P, Luco V, Sanhueza A, Oñate-Ponce A, Bolam JP, Henny P (2017) Characterization of the axon initial segment of mice substantia nigra dopaminergic neurons. J Comp Neurol 525:3529-3542.

Gowrishankar R, Hahn MK, Blakely RD (2014) Good riddance to dopamine: roles for the dopamine transporter in synaptic function and dopamineassociated brain disorders. Neurochem Int 73:42-48. 
Greenberg MM, Leitao C, Trogadis J, Stevens JK (1990) Irregular geometries in normal unmyelinated axons: a 3D serial EM analysis. J Neurocytol 19:978-988.

Groves PM, Linder JC, Young SJ (1994) 5-Hydroxydopamine-labeled dopaminergic axons: three-dimensional reconstructions of axons, synapses and postsynaptic targets in rat neostriatum. Neuroscience 58:593-604.

Hersch SM, Yi H, Heilman CJ, Edwards RH, Levey AI (1997) Subcellular localization and molecular topology of the dopamine transporter in the striatum and substantia nigra. J Comp Neurol 388:211-227.

Hillman GM, Schlessinger J (1982) Lateral diffusion of epidermal growth factor complexed to its surface receptors does not account for the thermal sensitivity of patch formation and endocytosis. Biochemistry 21:16671672.

Huang CY, Rasband MN (2018) Axon initial segments: structure, function, and disease. Ann NY Acad Sci 1420:46-61.

Iversen SD, Iversen LL (2007) Dopamine: 50 years in perspective. Trends Neurosci 30:188-193.

Jaber M, Jones S, Giros B, Caron MG (1997) The dopamine transporter: a crucial component regulating dopamine transmission. Mov Disord $12: 629-633$

Jung H, Gkogkas CG, Sonenberg N, Holt CE (2014) Remote control of gene function by local translation. Cell 157:26-40.

Kononenko NL, Claßen GA, Kuijpers M, Puchkov D, Maritzen T, Tempes A, Malik AR, Skalecka A, Bera S, Jaworski J, Haucke V (2017) Retrograde transport of TrkB-containing autophagosomes via the adaptor AP-2 mediates neuronal complexity and prevents neurodegeneration. Nat Commun 8:14819.

Leterrier C (2018) The axon initial segment: an updated viewpoint. J Neurosci 38:2135-2145.

Lin KJ, Lin KL, Chen SD, Liou CW, Chuang YC, Lin HY, Lin TK (2019) The overcrowded crossroads: mitochondria, alpha-synuclein, and the endolysosomal system interaction in Parkinson's. Int J Mol Sci 20:5312.

Luarte A, Cornejo VH, Bertin F, Gallardo J, Couve A (2018) The axonal endoplasmic reticulum: one organelle-many functions in development, maintenance, and plasticity. Dev Neurobiol 78:181-208.

Maday S, Holzbaur EL (2016) Compartment-specific regulation of autophagy in primary neurons. J Neurosci 36:5933-5945.

Matsuda W, Furuta T, Nakamura KC, Hioki H, Fujiyama F, Arai R, Kaneko $\mathrm{T}$ (2009) Single nigrostriatal dopaminergic neurons form widely spread and highly dense axonal arborizations in the neostriatum. J Neurosci 29:444-453.

Miranda M, Sorkina T, Grammatopoulos TN, Zawada WM, Sorkin A (2004) Multiple molecular determinants in the carboxyl terminus regulate dopamine transporter export from endoplasmic reticulum. J Biol Chem 279:30760-30770

Nirenberg MJ, Vaughan RA, Uhl GR, Kuhar MJ, Pickel VM (1996) The dopamine transporter is localized to dendritic and axonal plasma membranes of nigrostriatal dopaminergic neurons. J Neurosci 16:436-447.

Nirenberg MJ, Chan J, Vaughan RA, Uhl GR, Kuhar MJ, Pickel VM (1997) Immunogold localization of the dopamine transporter: an ultrastructural study of the rat ventral tegmental area. J Neurosci 17:5255-5262.

Paxinos G, Watson C (1998) The rat brain in stereotaxic coordinates, Ed 4. San Diego: Academic.

Perge JA, Koch K, Miller R, Sterling P, Balasubramanian V (2009) How the optic nerve allocates space, energy capacity, and information. J Neurosci 29:7917-7928

Pickel VM, Beckley SC, Joh TH, Reis DJ (1981) Ultrastructural immunocytochemical localization of tyrosine hydroxylase in the neostriatum. Brain Res 225:373-385.

Pissadaki EK, Bolam JP (2013) The energy cost of action potential propagation in dopamine neurons: clues to susceptibility in Parkinson's disease. Front Comput Neurosci 7:13.
Rahbek-Clemmensen T, Lycas MD, Erlendsson S, Eriksen J, Apuschkin M, Vilhardt F, Jørgensen TN, Hansen FH, Gether U (2017) Super-resolution microscopy reveals functional organization of dopamine transporters into cholesterol and neuronal activity-dependent nanodomains. Nat Commun 8:740.

Rahman AA, Morrison BE (2019) Contributions of VPS35 mutations to Parkinson's disease. Neuroscience 401:1-10.

Rao A, Richards TL, Simmons D, Zahniser NR, Sorkin A (2012) Epitopetagged dopamine transporter knock-in mice reveal rapid endocytic trafficking and filopodia targeting of the transporter in dopaminergic axons. FASEB J 26:1921-1933.

Rao A, Sorkin A, Zahniser NR (2013) Mice expressing markedly reduced striatal dopamine transporters exhibit increased locomotor activity, dopamine uptake turnover rate, and cocaine responsiveness. Synapse 67:668-677.

Ritchie JM (1982) On the relation between fibre diameter and conduction velocity in myelinated nerve fibres. Proc R Soc Lond B Biol Sci 217:29-35.

Sesack SR, Hawrylak VA, Matus C, Guido MA, Levey AI (1998) Dopamine axon varicosities in the prelimbic division of the rat prefrontal cortex exhibit sparse immunoreactivity for the dopamine transporter. J Neurosci 18:2697-2708

Shepherd GM, Raastad M, Andersen P (2002) General and variable features of varicosity spacing along unmyelinated axons in the hippocampus and cerebellum. Proc Natl Acad Sci USA 99:6340-6345.

Snyder SH (2002) Forty years of neurotransmitters: a personal account. Arch Gen Psychiatry 59:983-994.

Sorkina T, Richards TL, Rao A, Zahniser NR, Sorkin A (2009) Negative regulation of dopamine transporter endocytosis by membrane-proximal $\mathrm{N}$ terminal residues. J Neurosci 29:1361-1374.

Sorkina T, Caltagarone J, Sorkin A (2013) Flotillins regulate membrane mobility of the dopamine transporter but are not required for its protein kinase C dependent endocytosis. Traffic 14:709-724.

Sorkina T, Ma S, Larsen MB, Watkins SC, Sorkin A (2018) Small molecule induced oligomerization, clustering and clathrin-independent endocytosis of the dopamine transporter. Elife 7:e32293.

Surana S, Villarroel-Campos D, Lazo OM, Moretto E, Tosolini AP, Rhymes ER, Richter S, Sleigh JN, Schiavo G (2020) The evolution of the axonal transport toolkit. Traffic 21:13-33.

Tan PK, Waites C, Liu Y, Krantz DE, Edwards RH (1998) A leucine-based motif mediates the endocytosis of vesicular monoamine and acetylcholine transporters. J Biol Chem 273:17351-17360.

Veznedaroglu E, Milner TA (1992) Elimination of artifactual labeling of hippocampal mossy fibers seen following pre-embedding immunogold-silver technique by pretreatment with zinc chelator. Microsc Res Tech 23:100-101.

Volkow ND, Morales M (2015) The brain on drugs: from reward to addiction. Cell 162:712-725.

Wiley HS, Cunningham DD (1982) The endocytotic rate constant: a cellular parameter for quantitating receptor-mediated endocytosis. J Biol Chem 257:4222-4229.

Wise RA (2008) Dopamine and reward: the anhedonia hypothesis 30 years on. Neurotox Res 14:169-183.

Wu S, Fagan RR, Uttamapinant C, Lifshitz LM, Fogarty KE, Ting AY, Melikian HE (2017) The dopamine transporter recycles via a retromerdependent postendocytic mechanism: tracking studies using a novel fluorophore-coupling approach. J Neurosci 37:9438-9452.

Wu Y, Whiteus C, Xu CS, Hayworth KJ, Weinberg RJ, Hess HF, De Camilli P (2017) Contacts between the endoplasmic reticulum and other membranes in neurons. Proc Natl Acad Sci USA 114:E4859-E4867. 